\title{
Singularities of metrics on Hodge bundles and their topological invariants
}

\author{
Dennis Eriksson, Gerard Freixas i Montplet \\ and Christophe Mourougane \\ Dedicated to Christophe Soulé on the occasion of his 65th birthday
}

\begin{abstract}
We consider degenerations of complex projective Calabi-Yau varieties and study the singularities of $L^{2}$, Quillen and BCOV metrics on Hodge and determinant bundles. The dominant and subdominant terms in the expansions of the metrics close to non-smooth fibers are shown to be related to well-known topological invariants of singularities, such as limit Hodge structures, vanishing cycles and log-canonical thresholds. We also describe corresponding invariants for more general degenerating families in the case of the Quillen metric.
\end{abstract}

\section{Introduction}

In this article, we study the singularities of several natural metrics on combinations of Hodgetype bundles, for degenerating families of complex projective algebraic varieties. In particular, we provide topological interpretations of invariants associated with logarithmic singularities of these metrics. Our original motivation was a metrical approach to the canonical bundle formula for families of Calabi-Yau varieties [Kaw98, FM00, Amb04, Kol07]. The first instance of this formula goes back to Kodaira [Kod64, Theorem 12] and describes the relative canonical bundle of an elliptic surface in terms of a positive modular part and some topological invariants of the singular fibers. We were thus naturally led to the study of Hodge-type bundles, their metrics and behavior close to singular fibers.

As a matter of motivation, a classical example to keep in mind is the Hodge bundle $f_{*} \omega_{X / S}$ for a family of compact Riemann surfaces $f: X \rightarrow S$, endowed with its canonical $L^{2}$-metric or a Quillen metric on its determinant bundle (cf. Section 3.1). The latter topic is the main focus of the work of Bismut-Bost [BB90]. In the semi-stable case, they describe the singularities and the curvature current of the Quillen metric on the determinant of the Hodge bundle. In the special case where $S$ is the unit disk and there is a unique singular fiber $X_{0}$ at $0 \in S$, the principal part

Received 1 December 2016, accepted in final form 19 July 2017.

2010 Mathematics Subject Classification 14J32, 58K55, 58J52 (primary), 58K65, 14J70 (secondary).

Keywords: Hodge bundles, $L^{2}$-metric, Quillen metric, BCOV metric, log-canonical threshold, degeneracy index, limit Hodge structure, Milnor number, vanishing cycle.

This journal is (C) Foundation Compositio Mathematica 2018. This article is distributed with Open Access under the terms of the Creative Commons Attribution Non-Commercial License, which permits non-commercial reuse, distribution, and reproduction in any medium, provided that the original work is properly cited. For commercial re-use, please contact the Foundation Compositio Mathematica. 


\section{Metrics on Hodge Bundles}

of the curvature current is of the form

$$
\frac{\# \operatorname{sing}\left(X_{0}\right)}{12} \delta_{0}
$$

where $\delta_{0}$ is the Dirac current at 0 and \# $\operatorname{sing}\left(X_{0}\right)$ is the number of singular points in the fiber $X_{0}$.

In this article, we study analogues of this phenomenon for $L^{2}$-metrics on Hodge bundles for Calabi-Yau families (Theorem A), for Quillen metrics on determinant bundles (Theorem B) and for the so-called BCOV metric, which has found applications in mirror symmetry, for Calabi-Yau 3-folds (Theorem C).

To state our contributions, for the purpose of this introduction, we suppose that $f: X \rightarrow S$ is a flat, projective map of complex manifolds of relative dimension $n$, where $S$ is the unit disk with parameter $s$. We suppose the fibers $X_{s}=f^{-1}(s)$ connected and smooth for $s \neq 0$ (we say that $f$ is generically smooth). We also assume that $X$ carries a fixed Kähler metric. We denote the relative canonical bundle by $K_{X / S}:=K_{X} \otimes K_{S}^{-1}$.

Theorem A. Suppose that the general fiber of $f: X \rightarrow S$ is Calabi-Yau, that is, has a trivial canonical bundle. Let $\eta$ be a local holomorphic frame of the line bundle $f_{*} K_{X / S}$. Then if we define

we have

$$
\|\eta\|_{s}^{2}=\frac{1}{(2 \pi)^{n}}\left|\int_{X_{s}} \eta \wedge \bar{\eta}\right|
$$

$$
-\log \|\eta\|^{2}=\alpha \log |s|^{2}-\left.\beta \log |\log | s\right|^{2} \mid+O(1) \quad \text { as } \quad s \rightarrow 0,
$$

where $\alpha$ and $\beta$ have the following properties:

(i) The value of $\alpha$ is given by $\alpha=1-c_{X_{0}}(f) \in[0,1) \cap \mathbb{Q}$. Here $c_{X_{0}}(f)$ is the log-canonical threshold of $\left(X,-B, X_{0}\right)$ along $X_{0}$, where $B$ is the divisor of the evaluation map $f^{*} f_{*}\left(K_{X / S}\right) \rightarrow$ $K_{X / S}$. Moreover, $\exp (2 \pi i \alpha)$ is the eigenvalue of the semi-simple part of the monodromy acting on the graded piece $\mathrm{Gr}_{F}^{n} H_{\mathrm{lim}}^{n}$ of the middle limit Hodge structure of $X \rightarrow S$.

(ii) The integer $\beta=\delta\left(X, X_{0}\right) \in[0, n] \cap \mathbb{N}$ is the degeneracy index of $\left(X, X_{0}\right)$, computed through the geometry of the special fiber and $K_{X / S}$. Moreover, $\beta+n$ is the mixed Hodge structure weight of the 1-dimensional space $\mathrm{Gr}_{F}^{n} H_{\mathrm{lim}}^{n}$.

(iii) If $X \rightarrow S$ is birational to a model $Z \rightarrow S$, where $Z$ is normal with $K_{Z}$ locally free, and $Z_{0}$ has at worst canonical singularities, then $\alpha=\beta=0$ and the $L^{2}$-metric is continuous.

This statement summarizes the results in Section 2. Observe that the negative logarithm square of the norm is the potential of the first Chern form of the corresponding holomorphic Hermitian line bundle. On the smooth locus, the curvature of the $L^{2}$-metric is the Kähler form of the modular Weil-Petersson metric. Hence, Theorem A indicates the necessary correction of the Hodge bundle such that the $L^{2}$-metric becomes good in the sense of Mumford. A special case of the third point is morphisms with isolated ordinary quadratic singularities, in which case the $L^{2}$-metric is continuous.

Versions of Theorem A have already appeared in the work of other authors, in slightly different forms. The third point is proved by Wang in [Wan97, Proposition 2.3 with Corollary 1.2] (see also [RZ11, Theorem B.1] in the appendix by Gross) and in fact a converse is proven by Tosatti in [Tos15, Theorem 1.1]. As a special instance of canonical singularities, we mention the case of ordinary quadratic singularities when $n \geqslant 2$. The degeneracy index and log-canonical threshold 


\section{Eriksson, G. Freixas i Montplet and C. Mourougane}

have also been announced by Halle-Nicaise [HN12, Theorem 6.2.2] and detailed in [HN18, Theorem 3.3.3]. In the context of $\ell$-adic cohomology, they establish the analogous relationship as in the theorem above. There is also related work of Berman [Ber16, Section 3] on the asymptotics of $L^{2}$-metrics in terms of log-canonical thresholds. More recently, Boucksom-Jonsson [BJ17] studied asymptotics of volume forms in relationship with non-archimedean limits. Actually, the argument we provide for the asymptotics in terms of $c_{X_{0}}(f)$ and $\delta\left(X, X_{0}\right)$ is a specialization of the computations in [BJ17] and was communicated to us by S. Boucksom, whom we warmly thank.

In Sections 3 and 4, we shift our interest to the determinant line bundle endowed with a Quillen-type metric, instead of the direct image of the relative canonical bundle endowed with the $L^{2}$-metric. The main feature is that, after normalizing the metric, this bundle still detects the variation in moduli in its smooth part and has a degeneration mainly governed by the singular fibers and weakly depending on their germs of embedding. Now, suppose that $V$ is a Hermitian vector bundle on $X$, and let $\lambda(V)_{Q}$ be the determinant of the cohomology of $V$, equipped with the Quillen metric. This has a singularity at 0 , and our aim is to provide a topological measure of it. If $\sigma$ is a local holomorphic frame of $\lambda(V)$, then Yoshikawa [Yos07] proves, in our formulation,

$$
-\log \|\sigma\|_{\mathrm{Q}}^{2}=\left(\int_{X_{0}} \mathcal{Y}(X / S, V)\right) \log |s|^{2}+R(s) \quad \text { as } \quad s \rightarrow 0,
$$

where $\mathcal{Y}(X / S, V)$ is a certain cohomology class (Definition 3.6) and $R(s)$ is a continuous function of $s$. In this article, we study and generalize this class for families of varieties over a general parameter space $S$ whose total space $X$ is not necessarily smooth. This uses and underlines the Nash blowup instead of the Gauss morphism. The latter was actually introduced by Bismut [Bis97] and then further exploited by Yoshikawa [Yos07]. Our approach allows us to study the class $\mathcal{Y}(X / S, V)$ from the point of view of Fulton's intersection theory, which exhibits functoriality properties to the effect that we can use moduli space arguments in computations. For the formulation of the theorem, for simplicity, let $V$ be the trivial line bundle and set $Y(X / S)=\int_{X_{0}} \mathcal{Y}(X / S, V)$.

Theorem B. Suppose that $X \rightarrow S$ is a family over a disk, with $X$ not necessarily smooth.

(i) If $X \rightarrow S$ admits only isolated singularities in the special fiber $X_{0}$ and is locally a hypersurface in an $S$-smooth variety, then

$$
Y(X / S)=\frac{(-1)^{n+1}}{(n+2) !} \sum_{x \in X_{0}}\left(\mu_{X_{0}, x}+\mu_{X, x}\right),
$$

where $\mu_{X, 0}$ and $\mu_{X_{0}, 0}$ denote the Milnor numbers of the hypersurface singularities.

(ii) If $X$ is a family of hypersurfaces in $\mathbb{P}^{n}$ parametrized by $S$, then

$$
Y(X / S)=\frac{(-1)^{n+1}}{(n+2) !} \int_{X_{0}} c_{n+1}^{X_{0}}\left(\Omega_{X / S}\right),
$$

where $c_{n+1}^{X_{0}}\left(\Omega_{X / S}\right)$ denotes the localized top Chern class of $X \rightarrow S$.

(iii) If $X \rightarrow S$ is a family of $K 3$ or abelian surfaces, with $X$ smooth and $K_{X}$ trivial, the same formula holds. One can conclude that

$$
Y(X / S)=\frac{-1}{24}\left(\chi\left(X_{\infty}\right)-\chi\left(X_{0}\right)\right) .
$$

In fact, for general families $X \rightarrow S$ with $X$ smooth, we have the fundamental relation

$$
\int_{X_{0}} c_{n+1}^{X_{0}}\left(\Omega_{X / S}\right)=(-1)^{n}\left(\chi\left(X_{\infty}\right)-\chi\left(X_{0}\right)\right) .
$$




\section{Metrics on Hodge Bundles}

The expression $\chi\left(X_{\infty}\right)-\chi\left(X_{0}\right)$ is the total dimension of the vanishing cycles of the family, that is, the difference between the topological Euler characteristic of the special fiber $X_{0}$ and that of a general fiber $X_{\infty}$.

The developments abutting to Theorem B are the object of Section 3. We stress here that the intersection-theoretic approach is well suited to other geometric settings. For instance, in the "arithmetic situation" (that is, $S$ is the spectrum of a discrete valuation ring of mixed characteristic), the Yoshikawa class can still be defined and may be seen as a discriminant, meaning a measure of bad reduction. An example of this principle was studied by the first author in [Eri16] and applied in the study of Quillen metrics on degenerating Riemann surfaces [Eri13]. This was a source of inspiration for the present work.

In Section 4, we turn our attention to a particular combination of Hodge-type bundles. For a smooth family $f: X \rightarrow S$, one can consider the vector bundles $R^{q} f_{*} \Omega_{X / S}^{p}$ coming from the Hodge filtration on relative de Rham cohomology. Taking weighted determinants of these vector bundles, one introduces the BCOV line bundle (named after Bershadsky-Cecotti-OoguriVafa [BCOV94])

$$
\lambda_{\mathrm{BCOV}}=\bigotimes_{p=0}^{n} \lambda\left(\Omega_{X / S}^{p}\right)^{(-1)^{p} p}=\bigotimes_{p, q=0}^{n} \operatorname{det}\left(R^{q} f_{*} \Omega_{X / S}^{p}\right)^{(-1)^{p+q} p} .
$$

Following Fang-Lu-Yoshikawa [FLY08], after a suitable rescaling of the Quillen metric on $\lambda_{\mathrm{BCOV}}$, one defines the BCOV metric. For a family of Calabi-Yau varieties, this is independent of the initially chosen Kähler metric, and its curvature is given by the modular Weil-Petersson form. Therefore, it is an intrinsic invariant of the family. As [FLY08] illustrates, for applications to mirror symmetry in physics, it is important to determine the singularities of the BCOV metric under degeneration. Hence, let us now assume that $f: X \rightarrow S$ is only generically smooth. The line bundle $\lambda_{\mathrm{BCOV}}$ (initially defined on the smooth locus) has a natural extension to $S$, called the Kähler extension, which we denote by $\widetilde{\lambda}_{\mathrm{BCOV}}$. Then, the BCOV metric on $\lambda_{\mathrm{BCOV}}$ can be seen as a singular metric on $\widetilde{\lambda}_{\mathrm{BCOV}}$. The last statement of this introduction summarizes our results on the singularities of the BCOV metric on $\widetilde{\lambda}_{\mathrm{BCOV}}$.

Theorem C. Suppose that $K_{X}$ is trivial. Let $\eta$ be a local holomorphic frame of $\widetilde{\lambda}_{\mathrm{BCOV}}$.

(i) The asymptotic expansion of the BCOV metric is

$$
-\log \|\eta\|_{\mathrm{BCOV}}^{2}=\alpha_{\mathrm{BCOV}} \log |s|^{2}-\left.\frac{\chi\left(X_{\infty}\right)}{12} \beta \log |\log | s\right|^{2} \mid+O(1) \quad \text { as } \quad s \rightarrow 0 .
$$

Here

$$
\alpha_{\mathrm{BCOV}}=-\frac{9 n^{2}+11 n+2}{24}\left(\chi\left(X_{\infty}\right)-\chi\left(X_{0}\right)\right)+\frac{\alpha}{12} \chi\left(X_{\infty}\right),
$$

and $\alpha$ and $\beta$ are as in Theorem A. In particular, $\alpha_{\mathrm{BCOV}}$ is expressed in terms of vanishing cycles and the topological Euler characteristic of a general fiber.

(ii) If the monodromy action on $H_{\lim }^{n}$ is unipotent (for example, if $f$ is semi-stable), then $\alpha_{\mathrm{BCOV}}$ further simplifies to

$$
\alpha_{\mathrm{BCOV}}=-\frac{9 n^{2}+11 n+2}{24}\left(\chi\left(X_{\infty}\right)-\chi\left(X_{0}\right)\right) .
$$

(iii) If $f$ has only isolated ordinary quadratic singularities and $n \geqslant 2$, then

$$
\alpha_{\mathrm{BCOV}}=(-1)^{n+1} \frac{9 n^{2}+11 n+2}{24} \# \operatorname{sing}\left(X_{0}\right)
$$




\section{Eriksson, G. Freixas i Montplet and C. Mourougane}

so that

$$
-\log \|\eta\|_{\mathrm{BCOV}}^{2}=(-1)^{n+1} \frac{9 n^{2}+11 n+2}{24} \# \operatorname{sing}\left(X_{0}\right) \log |s|^{2}+O(1) .
$$

Such families with trivial canonical bundle are commonly known as Kulikov families, named after work of Kulikov on semi-stable degenerations of K3 surfaces [Kul77]. Examples in other dimensions are known to exist [KN94, Lee10]. Another situation of Kulikov families is when $f$ has relative dimension $n \geqslant 2$ and presents only isolated singularities, so that the Kulikov assumption in the third point of Theorem $\mathrm{C}$ is automatic. In fact, we provide a general closed formula for the logarithmic divergence, without any assumption on $K_{X}$. In any event, Theorem $\mathrm{C}$ describes the necessary correction to the BCOV metric on $\widetilde{\lambda}_{\mathrm{BCOV}}$ in order to obtain a Mumford-good Hermitian metric.

The expression of $\alpha_{\mathrm{BCOV}}$ in part (iii) for ordinary quadratic singularities was first observed by Yoshikawa (private communication with the authors). Our approach is based on independent ideas, relying on the general expression in part (i) and the fact that $\alpha=\beta=0$ for these types of singularities.

\section{Degeneration of $L^{2}$-metrics on the Hodge bundle}

\subsection{Background on the Hodge bundle for Calabi-Yau and Kulikov families}

Let $f: X \rightarrow S$ be a proper flat morphism with connected fibers of dimension $n$ from a complex manifold $X$ to a smooth complex curve $S$. We will refer to such a map as a family. Assume that $f$ is generically smooth (or submersive) with respect to the Zariski topology. The relative cotangent sheaf $\Omega_{X / S}$ (or sheaf of relative Kähler differentials) then fits into a short exact sequence

$$
0 \rightarrow f^{*} \Omega_{S} \stackrel{d f^{t}}{\longrightarrow} \Omega_{X} \rightarrow \Omega_{X / S} \rightarrow 0 .
$$

The exactness on the left is guaranteed by the generic smoothness assumption. The relative canonical bundle is defined to be

$$
K_{X / S}:=K_{X} \otimes f^{*} K_{S}^{-1} .
$$

It coincides with $\Lambda^{n} \Omega_{X / S}$ at the points where $f$ is submersive.

Now, assume that the smooth fibers of $f$ have trivial canonical bundle. Then the direct image sheaf $f_{*}\left(K_{X / S}\right)$, the Hodge bundle, is locally free of rank 1. Indeed, it is a torsion-free sheaf on a smooth curve, and hence locally free. Moreover, on a Zariski-dense open subset of $S$, it has rank 1. The evaluation map of line bundles

$$
\text { ev : } f^{*} f_{*}\left(K_{X / S}\right) \rightarrow K_{X / S}
$$

is an isomorphism over smooth fibers, by base change, and is injective. We denote its zero divisor by $B$. By construction, $B$ is a divisor supported in the singular fibers of $f$ and depends on the model $X$. The injectivity of the evaluation map implies that $B$ is effective. With this notation, we have the relation

$$
K_{X / S}=f^{*} f_{*}\left(K_{X / S}\right) \otimes \mathcal{O}_{X}(B) .
$$

We observe that the (local) relative canonical divisor $B$ cannot contain any full fibers of $f$. For this, let $s$ be a local parameter on the curve $S$ centered at a point 0 . Let $\eta$ be a local section of $f_{*}\left(K_{X}\right)$ on an open set $U$ of $S$, not divisible by $s$ in $f_{*}\left(K_{X}\right)$. If the zero divisor $B$ of ev contained the whole fiber $X_{0}$, then $\left(\mathrm{ev} f^{*} \eta\right) / s$ would be a form in $K_{X}\left(f^{-1}(U)\right)$. But it is not of 


\section{Metrics on Hodge Bundles}

the form ev $f^{*} \mu$ for some $\mu$ in $f_{*}\left(K_{X}\right)(U)$, which contradicts the surjectivity of ev on the open tube $\left(f^{-1} U\right)$.

In the case where $B$ is trivial, we call $f: X \rightarrow S$ a Kulikov family. Kulikov models are in general difficult to describe. For families of K3 surfaces, Kulikov [Kul77] established the existence of such models in the semi-stable case. In arbitrary dimension, examples are obtained by the smoothing of suitable normal crossings varieties [KN94, Lee10]. Finally, we remark that if the special fiber has at least two components, their intersection is part of the $f$-singular locus $Z$ and any component therein is of dimension at least $n+1-2=n-1$. Hence, if $Z$ is of dimension at most $n-2$, then any singular fiber $X_{0}$ is necessarily irreducible and reduced. We infer that $B$ is empty in this case. A particular instance of this fact is given by morphisms of relative dimension $n \geqslant 2$ and isolated singularities.

\subsection{Log-canonical threshold, degeneracy index and the singularities of the $L^{2}$-metric}

Let $f: X \rightarrow S$ be a generically smooth family between complex manifolds, of relative dimension $n$, where $S$ is a curve. Assume that the smooth fibers of $f$ have a trivial canonical bundle, so that $f_{*}\left(K_{X / S}\right)$ is a line bundle. On the smooth locus in $S$, this line bundle affords an intrinsic $L^{2}$, or Hodge, metric. If $\eta$ is a non-vanishing $n$-form on a smooth fiber $X_{s}$, then

$$
\|\eta\|_{L^{2}, s}^{2}=\frac{1}{(2 \pi)^{n}}\left|\int_{X_{s}} \eta \wedge \bar{\eta}\right|
$$

If $\eta$ extends to a trivialization of $f_{*}\left(K_{X / S}\right)$ in a neighborhood of $s$, then $\|\eta\|_{L^{2}, s}$ changes smoothly with $s$. The question is to analyze the behavior of the $L^{2}$-metric close to the singular locus of $f$ in $S$. For the sake of simplicity, from here on, we assume that $S$ is a disk centered at 0 and that there is at most a singular fiber at 0 .

The formation of $f_{*}\left(K_{X / S}\right)$ is invariant under blowups along regular centers in the special fiber. Therefore, for the purpose of analyzing the $L^{2}$-metric, we may assume (after a Hironaka resolution) that the singular fiber of $f: X \rightarrow S$ decomposes into irreducible components $a_{j} E_{j}$, with $E_{j}$ smooth, meeting with normal crossings

$$
X_{0}=\sum a_{j} E_{j} .
$$

We write the zero divisor of the evaluation map (2.2) in the form $B=\sum\left(b_{j}-1\right) E_{j}$. Following Kollár [Kol97, Section 8, especially Definition 8.1] (see also Berman [Ber16, Section 3.4, especially Proposition 3.8]), we define the log-canonical threshold of $\left(X,-B, X_{0}\right)$ along $X_{0}$ by

$$
c_{X_{0}}\left(X,-B, X_{0}\right)=\min _{j}\left(\frac{b_{j}}{a_{j}}\right) .
$$

As in [Kol97], we will allow the abuse of notation $c_{X_{0}}(f)$ for $c_{X_{0}}\left(X,-B, X_{0}\right)$. In addition, we define

$$
b\left(X, X_{0}\right):=\max \left\{\sharp J \mid \cap_{J} E_{j} \neq \emptyset \text { and } \forall j \in J, \frac{b_{j}}{a_{j}}=c_{X_{0}}\left(X,-B, X_{0}\right)\right\} .
$$

Notice that $b\left(X, X_{0}\right)-1$ is the degeneracy index $\delta\left(X, X_{0}\right)$ defined by Halle-Nicaise [HN12, Definition 6.2.1].

The log-canonical threshold and the degeneracy index govern the asymptotic of the $L^{2}$-metric close to the singular locus.

Proposition 2.1. Let $\eta$ be a holomorphic frame of $f_{*} K_{X / S}$. Then the $L^{2}$-metric on $f_{*} K_{X / S}$ 


\section{Eriksson, G. Freixas i Montplet and C. Mourougane}

degenerates as

$$
-\log \|\eta\|_{L^{2}}^{2}=\left(1-c_{X_{0}}(f)\right) \log |s|^{2}-\left.\left(b\left(X, X_{0}\right)-1\right) \log |\log | s\right|^{2} \mid+O(1) \quad \text { as } \quad s \rightarrow 0,
$$

where $s$ is the local coordinate on $S$.

Proof. The isomorphism $K_{X / S}=\operatorname{det} \Omega_{X / S}=\operatorname{det}\left(\Omega_{X} / f^{*} \Omega_{S}\right)$ on the smooth part of $f$ yields a description of the map

$$
f^{*} K_{S} \otimes K_{X / S} \rightarrow K_{X}, \quad f^{*} d s \otimes[u] \mapsto f^{*} d s \wedge u
$$

Rewrite the relation $(2.3)$ as

$$
f^{*} K_{S} \otimes f^{*} f_{*} K_{X / S}=\mathcal{O}_{X}(-B) \otimes K_{X} .
$$

Choose a point $x_{0} \in X_{0}$. Set $J\left(x_{0}\right):=\left\{j \mid x_{0} \in E_{j}\right\}$. Choose local coordinates $\left(z_{1}, z_{2}, \ldots, z_{n+1}\right)$ on $X$ centered at $x_{0}$ such that for $j \in J\left(x_{0}\right)$, the divisor $E_{j}$ is given by $z_{j}=0$ and the map $f$ can be written, locally around $x_{0}$, as

$$
f:\left(z_{1}, z_{2}, \ldots, z_{n+1}\right) \mapsto s=\prod_{j \in J\left(x_{0}\right)} z_{j}^{a_{j}} .
$$

The isomorphism (2.4) shows the existence of an open covering $\left(U_{\alpha}\right)$ of $X$ by coordinate charts and invertible holomorphic functions $f_{\alpha}$ such that on $U_{\alpha}$,

$$
f^{*} d s \wedge \operatorname{ev}(\eta)=f_{\alpha} \prod_{j \in J\left(x_{0}\right)} z_{j}^{b_{j}-1} d z_{1} \wedge d z_{2} \wedge \cdots \wedge d z_{n+1} .
$$

We choose a partition of unity $\left(\phi_{\alpha}\right)$ built from an open covering of $X$ where the previous simplifications hold. Choose a $j_{0} \in J\left(x_{0}\right)$ such that $b_{j_{0}} / a_{j_{0}}=\min _{J\left(x_{0}\right)}\left(b_{j} / a_{j}\right)$, and note that

$$
d z_{1} \wedge d z_{2} \wedge \cdots \wedge d z_{n+1}=(-1)^{j_{0}} \frac{z_{j_{0}}}{a_{j_{0}}} f^{*} \frac{d s}{s} \wedge d z_{1} \wedge d z_{2} \wedge \cdots \wedge d z_{j_{0}-1} \wedge d z_{j_{0}+1} \wedge \cdots \wedge d z_{n+1} .
$$

We introduce the change of variables $z_{j}=e^{\rho_{j} / a_{j}} e^{i \theta_{j}}$ for $j \in J\left(x_{0}\right)$ and $z_{k}=r_{k} e^{i \theta_{k}}$ for $k \in$ $\{1, \ldots, n+1\}-J\left(x_{0}\right)$. The set of integration is defined by $\left|z_{i}\right| \leqslant 1$ and $\prod_{j \in J\left(x_{0}\right)} z_{j}^{a_{j}}=s$, in other words, by $\rho_{j} \leqslant 0,0 \leqslant r_{k} \leqslant 1$, and $\sum_{j \in J\left(x_{0}\right)} a_{j} \theta_{j}=\arg (s)$ and $\sum_{j \in J\left(x_{0}\right)} \rho_{j}=\log |s|$. We have

$$
\begin{aligned}
& \left\|\phi_{\alpha} \eta_{s}\right\|_{L^{2}\left(X_{s}\right)}^{2}=\frac{f_{\star}\left(\phi_{\alpha} f^{*}(i d s \wedge d \bar{s}) \wedge i^{n^{2}} \mathrm{ev}(\eta) \wedge \overline{\mathrm{ev}(\eta)}\right)}{i d s \wedge d \bar{s}} \\
& =\frac{1}{(2 \pi)^{n}|s|^{2}} \int_{X_{s}} \phi_{\alpha} \frac{\left|f_{\alpha}(z)\right|^{2}}{\left|a_{j_{0}}\right|^{2}} \prod_{j \in J\left(x_{0}\right)}\left|z_{j}\right|^{2 b_{j}-2}\left|z_{j_{0}}\right|^{2}\left|d z_{1}\right|^{2}\left|d z_{2}\right|^{2} \cdots\left|d z_{j_{0}-1}\right|^{2}\left|d z_{j_{0}+1}\right|^{2} \cdots\left|d z_{n+1}\right|^{2} \\
& =\frac{1}{(2 \pi)^{n}|s|^{2}} \int_{X_{s}} \phi_{\alpha} \frac{\left|f_{\alpha}(z)\right|^{2}}{\prod_{j \in J\left(x_{0}\right)}\left|a_{j}\right|^{2}} \prod_{j \in J\left(x_{0}\right)} e^{\left(2 b_{j} / a_{j}\right) \rho_{j}} \prod_{j \in J\left(x_{0}\right)-j_{0}} d \theta_{j} d \rho_{j} \prod_{k \in\{1, \ldots, n+1\}-J\left(x_{0}\right)} r_{k} d r_{k} d \theta_{k} \\
& \equiv C|s|^{2\left(b_{j_{0}} / a_{j_{0}}-1\right)} \int_{\sum \rho_{j}=\log |s|} \phi_{\alpha}\left|f_{\alpha}(z)\right|^{2} \prod_{j \in J\left(x_{0}\right)} e^{\left(2 b_{j} / a_{j}-2 b_{j_{0}} / a_{j_{0}}\right) \rho_{j}} d \rho_{1} d \rho_{2} \cdots d \rho_{j_{0}-1} d \rho_{j_{0}+1} \cdots d \rho_{n+1} .
\end{aligned}
$$

Adding those estimates for the different $\alpha$ and neglecting bounded terms, we derive the desired estimate

$$
-\log \left\|\eta_{s}\right\|_{L^{2}}^{2}=\left(1-\min _{j}\left(\frac{b_{j}}{a_{j}}\right)\right) \log |s|^{2}-\left.\sharp\left\{j \neq j_{0} \mid x_{0} \in E_{j} \text { and } \frac{b_{j}}{a_{j}}=\frac{b_{j_{0}}}{a_{j_{0}}}\right\} \log |\log | s\right|^{2} \mid+O(1) .
$$




\section{Metrics on Hodge Bundles}

Remark 2.2. Now, consider the particular case where $X \rightarrow S$ is semi-stable. Then $a_{i}=1$ for all $i$ and since the divisor $B$ does not contain a whole fiber (see Section 2.1), there is at least one index $i$ with $b_{i}=1$. We conclude that $c_{X_{0}}(f)=\min _{j}\left(b_{j} / a_{j}\right)=1$. If the family is moreover a Kulikov model, then $b_{i}=1$ for all $i$. In this case, it follows that $b\left(X, X_{0}\right)$ is simply the maximal number of intersecting components in the special fiber.

\subsection{The $L^{2}$-metric and semi-stable reduction}

Let us now examine the change of the $L^{2}$-metric under semi-stable reduction. We consider a semistable reduction diagram

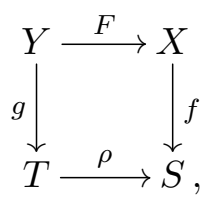

where $g$ is a semi-stable family, $\rho$ is the finite morphism $t \mapsto s=t^{e}$ and $F$ a generically finite morphism. From [MT09, Lemmas 3.3 and 4.2], we know that $\left(g_{*}\left(K_{Y / T}\right), L^{2}\right)$ embeds isometrically into $\left(\rho^{*} f_{*}\left(K_{X / S}\right), \rho^{*} L^{2}\right)$. A local frame $\xi$ for $g_{*}\left(K_{Y / T}\right)$ hence relates to a local frame $\eta$ for $f_{*}\left(K_{X / S}\right)$ through

$$
\xi=t^{a} \rho^{*} \eta
$$

where $a$ can be recovered by the formula

$$
a=\operatorname{dim}_{\mathbb{C}}\left(\frac{\rho^{*} f_{*}\left(K_{X / S}\right)}{g_{*}\left(K_{Y / T}\right)}\right)_{0} .
$$

From the previous proposition, we get the following statement.

Proposition 2.3. The asymptotic of the $L^{2}$-metric on $f_{*}\left(K_{X / S}\right)$ is of the shape

$$
-\log \|\eta\|^{2}=\alpha \log |s|^{2}-\left.\beta \log |\log | s\right|^{2} \mid+C+O\left(\frac{1}{\log |s|}\right),
$$

where

$$
\alpha=\frac{a}{e}=\frac{1}{e} \operatorname{dim}_{\mathbb{C}}\left(\frac{\rho^{*} f_{*}\left(K_{X / S}\right)}{g_{*}\left(K_{Y / T}\right)}\right)_{0} \quad \text { and } \quad \beta=b\left(X, X_{0}\right)-1=b\left(Y, Y_{0}\right)-1 .
$$

Remark 2.4. The fact that the metric has the above shape, with $\alpha=0$ in the semi-stable case, is already stated in [Yos10, Theorem 6.8].

\subsection{The $L^{2}$-metric via variation of Hodge structures}

Let $f: X \rightarrow \Delta$ be a proper Kähler morphism with connected fibers of dimension $n$ from a complex manifold $X$ to the complex unit disk $\Delta$, which is a holomorphic submersion on $\Delta^{\times}$. We suppose that the special fiber is a normal crossings divisor and that the equation for $f$ is locally given by $s=z_{1}^{n_{1}} \cdots z_{k}^{n_{k}}$, where $s$ is the standard parameter on $\Delta$. Denote the smooth part of $f$ by $f^{\times}:=f^{-1}\left(\Delta^{\times}\right) \rightarrow \Delta^{\times}$. Let $\gamma$ be the monodromy operator of the local system $R^{n} f_{*}^{\times} \mathbb{C}$, and let $\gamma=\gamma_{u} \gamma_{s}=\gamma_{s} \gamma_{u}$ be its Jordan decomposition, where $\gamma_{u}$ is unipotent and $\gamma_{s}$ semi-simple.

The aim of this section is to prove the following statement.

Proposition 2.5. With the previous notation, suppose furthermore that $h^{n, 0}=1$. Then,

(i) $\exp (2 \pi i \alpha)=\exp \left(-2 \pi i c_{X_{0}}(f)\right)$ is the eigenvalue of $\gamma_{s}$ acting on $\operatorname{Gr}_{F}^{n} H_{\text {lim }}^{n}=F^{n} H_{\text {lim }}^{n}$; 


\section{Eriksson, G. Freixas i Montplet and C. Mourougane}

(ii) $n+\beta$ is the weight of the 1-dimensional space $\operatorname{Gr}_{F}^{n} H_{\text {lim }}^{n}$.

Remark 2.6. The result seems to be known and is announced in [HN12, Theorem 6.2.2(2)] and detailed in [HN18, Theorem 3.3.3]. The authors of those papers have informed us that the methods amount to the usage of Steenbrink's constructions of the logarithmic relative de Rham complex. Our method of proof is based on a (nowadays standard) combination of Deligne extensions of local systems and Schmid's construction of the limit mixed Hodge structure.

Proof. Denote the differentiable manifold underlying a general fiber of $f$ by $X_{\infty}$. We will use the correspondence between an element $\mathbf{Q}$ in $H^{n}\left(X_{\infty}, \mathbb{C}\right)$ and the corresponding multi-valued flat section $Q$ of the local system $R^{n} f_{*}^{\times} \mathbb{C}$. Let $\rho=\exp (2 \pi i-): \mathbb{H} \rightarrow \Delta^{\times}$be the universal covering of the punctured unit disk, and for $\tau \in \mathbb{H}$, set $s=\exp (2 \pi i \tau)$. Set $\Gamma=N+S$ with $N=(1 / 2 \pi i) \log \gamma_{u}$ and $S=(1 / 2 \pi i) \log \gamma_{s}$, where for $S$, we have fixed the branch of the logarithm having imaginary part in $[0,2 \pi)$. Hence, $S$ has eigenvalues in $[0,1)$.

Let $\mathbf{f}_{1}, \ldots, \mathbf{f}_{N}$ be a basis of $H^{n}\left(X_{\infty}, \mathbb{C}\right)$. The corresponding multi-valued flat basis satisfies $f_{i}(\tau+1)=\gamma f_{i}(\tau)$. If we define the twisted sections

$$
e_{i}:=s^{-\Gamma} f_{i}=\exp (-2 \pi i \tau \Gamma) f_{i},
$$

then we have $e_{i}(\tau+1)=e_{i}(\tau)$. The Deligne canonical extension $\mathcal{H}^{n}$ of $R^{n} f_{*}^{\times} \mathbb{C} \otimes \mathcal{O}_{\Delta^{\times}}$, also called the upper extension due to the choice of the logarithm, is defined to be the locally free $\mathcal{O}_{\Delta}$ module generated by the $e_{i}$. The Gauss-Manin connection on $R^{n} f_{*}^{\times} \mathbb{C} \otimes \mathcal{O}_{\Delta} \times$ extends to a regular singular connection on $\mathcal{H}^{n}$. Its residue is readily computed in the basis $\left(e_{i}\right)$ and seen to coincide with $-\Gamma$.

We denote by $H_{\text {lim }}^{n}$ the limit (mixed) Hodge structure on $H^{n}\left(X_{\infty}, \mathbb{C}\right)$, the cohomology of a general fiber. By construction, $H_{\lim }^{n}$ is equipped with a decreasing filtration $F^{p} H^{n}\left(X_{\infty}, \mathbb{C}\right)$, the Hodge filtration, and an increasing filtration $W_{k} H^{n}\left(X_{\infty}, \mathbb{C}\right)$, the weight filtration built from the nilpotent operator $N$. Moreover, $H_{\text {lim }}^{n}$ may be identified with the fiber of $\mathcal{H}^{n}$ at 0 , with monodromy action given in terms of the residue $\exp (2 \pi i \Gamma)$ of the Gauss-Manin connection.

By the nilpotent orbit theorem [Sch73], and as shown by Kawamata [Kaw82, Lemma 1],

$$
f_{*}\left(K_{X / \Delta}\right)=\iota_{*} f_{*}^{\times}\left(K_{X^{\times} / \Delta^{\times}}\right) \cap \mathcal{H}^{n},
$$

where $\iota: \Delta^{\times} \rightarrow \Delta$ is the inclusion. We can therefore write a local frame $\eta$ for $f_{*}\left(K_{X / \Delta}\right)$ as

$$
\eta=\sum_{i} \eta_{i}(s) e_{i}(s)=\sum_{i} \eta_{i}(s) \exp (-2 \pi i \tau \Gamma) f_{i}
$$

where the $\eta_{i}$ are local holomorphic functions. In this case, the corresponding limit of the twisted period is $\mathbf{Q}_{\infty}:=\sum_{i} \eta_{i}(0) \mathbf{e}_{i} \in F^{n} H_{\mathrm{lim}}^{n}$.

Let $\ell$ be the integer such that $\mathbf{Q}_{\infty}$ belongs to $W_{\ell}$ but not to $W_{\ell-1}$. By the construction of the weight filtration, the nilpotent operator $N$ maps $W_{\ell}$ to $W_{\ell-2}$. The semi-simple part $\gamma_{s}$ (and hence $S$ ) acts on $H_{\text {lim }}^{n}$ as a mixed Hodge structure operator [Ste77, Theorem 2.13]. Write $\omega_{j}:=\exp \left(2 \pi i \lambda_{j}\right)$, where $\lambda_{j}$ is a non-increasing sequence of rational numbers in $[0,1)$, for the sequence of eigenvalues of $\gamma_{s}$ acting on $W_{\ell} / W_{\ell-1}$. Choose a basis $\left(\mathbf{e}_{j}\right)$ of $H_{\lim }^{n}$ adapted to the filtration $W$. Hence, $\mathbf{Q}_{\infty}$ can be decomposed as

$$
\mathbf{Q}_{\infty}=\mathbf{Q}^{+}+\mathbf{Q}^{\prime}
$$

where $\mathbf{Q}^{+}:=\sum_{j} q_{j} \mathbf{e}_{j}, S \mathbf{e}_{j}=\lambda_{j} \mathbf{e}_{j}+\mathbf{e}_{j}^{\prime}$ and $\mathbf{Q}^{\prime}$ and the $\mathbf{e}_{j}^{\prime}$ belong to $W_{\ell-1}$. As $\gamma_{s}$ respects the Hodge filtration on $H^{n}\left(X_{\infty}, \mathbb{C}\right)$, and as $h^{n, 0}=1$, we know that $F^{n} H^{n}\left(X_{\infty}, \mathbb{C}\right)$ is an eigenspace 


\section{Metrics on Hodge Bundles}

for $S$, with eigenvalue, say, $\lambda$. From the freeness of $\left(\mathbf{e}_{j}\right)$, it follows that for each $j$, either $q_{j}=0$ or $\lambda_{j}=\lambda$, so that

$$
S\left(\mathbf{Q}^{+}\right)=\lambda\left(\mathbf{Q}^{+}\right)+\mathbf{Q}^{\prime \prime}
$$

where $\mathbf{Q}^{\prime \prime} \in W_{\ell-1}$.

We denote the intersection form on $R^{n} f_{*}^{\times} \mathbb{C}$ by $I$ and let $C$ be the Weil operator such that $I(C v, \bar{v})$ is positive. As the coefficients $\eta_{i}$ are holomorphic,

$$
\int_{X_{s}} i^{n^{2}} \eta(s) \wedge \overline{\eta(s)}=I(C \eta(s), \overline{\eta(s)})=I\left(C e^{-2 \pi i \tau \Gamma} Q_{\infty}(s), \overline{e^{-2 \pi i \tau \Gamma} Q_{\infty}(s)}\right)(1+O(|s|)) .
$$

By the SL(2)-orbit theorem [Sch73, Theorem 6.6], that gives the asymptotic of the orbit of elements in $W_{\bullet}$, the leading contribution comes from elements in $W_{\ell}$ not in $W_{\ell-1}$ :

$$
\begin{aligned}
I\left(C e^{-2 \pi i \tau \Gamma} Q_{\infty}(s), \overline{e^{-2 \pi i \tau \Gamma} Q_{\infty}(s)}\right) & =I\left(C e^{-2 \pi i \tau \Gamma} Q^{+}(s), \overline{e^{-2 \pi i \tau \Gamma} Q^{+}(s)}\right)\left(1+O\left(\operatorname{Im}(\tau)^{-1}\right)\right) \\
& =|s|^{-2 \lambda} I\left(C e^{-2 \pi i \tau N} Q^{+}, \overline{e^{-2 \pi i \tau N} Q^{+}}\right)\left(1+O\left(\operatorname{Im}(\tau)^{-1}\right)\right) .
\end{aligned}
$$

Now, for the principal nilpotent orbit $\eta^{+}(s):=e^{-2 \pi i \tau N} Q^{+}$, the quantity

$$
I\left(C \eta^{+}(s), \overline{\eta^{+}(s)}\right)=I\left(C e^{-2 \pi i \tau N} Q^{+}, \overline{e^{-2 \pi i \tau N} Q^{+}}\right)=I\left(C e^{4 \pi \operatorname{Im}(\tau) N} Q^{+}, \overline{Q^{+}}\right)
$$

is a polynomial $P(\operatorname{Im}(\tau))$ of degree $\mu$ in $\operatorname{Im}(\tau)$, whose leading term is

$$
\frac{(4 \pi)^{\mu} \operatorname{Im}(\tau)^{\mu}}{\mu !} I\left(C N^{\mu} Q^{+}, \overline{Q^{+}}\right)
$$

(compare with [Wan97, Section 1]). The degree $\mu$ is the order of the nilpotent operator $N$ acting on $\mathbf{Q}^{+}$. Hence, by the polarized condition [CK82, (2.10)], and because $\mathbf{Q}_{\infty}$ and $\mathbf{Q}^{+}$differ by an element in $W_{\ell-1}$, it is exactly the order of the nilpotent monodromy operator $N$ acting on the limit twisted period $\mathbf{Q}_{\infty}$.

The asymptotic of the $L^{2}$-norm is therefore $-\log \|\eta(s)\|_{L^{2}}^{2} \simeq \lambda \log |s|^{2}-\left.\mu \log |\log | s\right|^{2} \mid$.

Remark 2.7. In the unipotent case, and with the notation as in the proof of Proposition 2.5, from $\|\eta(s)\|_{L^{2}}^{2}=P(-(1 / 2 \pi) \log |s|)+\rho_{1}(\tau)$, we infer that the curvature of $\left(\pi_{*}\left(K_{X / S}\right), L^{2}\right)$ (that is, the Weil-Petersson metric) has Poincaré growth

$$
d d^{c}\left(-\log \|\eta(\tau)\|_{L^{2}}^{2}\right)=\left(\frac{\left(P^{\prime}\right)^{2}-P P^{\prime \prime}+\rho_{2}(z)}{P^{2}+\rho_{3}(\tau)}\right) i d \tau \wedge d \bar{\tau} \simeq\left(\frac{\mu}{(\operatorname{Im} \tau)^{2}}+\rho_{4}(\tau)\right) i d \tau \wedge d \bar{\tau},
$$

where the $\rho$ are functions which, together with all their derivatives, exponentially decrease to zero as $\operatorname{Im}(\tau)$ tends to $+\infty$, with rate of decay independent of $\operatorname{Re}(\tau)$.

Recall that a variety $Z$ has canonical singularities if $Z$ is normal and the canonical divisor $K_{Z}$ is $\mathbb{Q}$-Cartier, and if for any resolution of singularities $\mu: Z^{\prime} \rightarrow Z$, the divisor $K_{Z^{\prime}}-\mu^{*} K_{Z}$ is effective. It follows that if $K_{Z}$ is Cartier, then $\mu_{*} K_{Z^{\prime}} \simeq K_{Z}$. If $g: Z \rightarrow \Delta$ is such that $Z_{0}$ has canonical singularities, then so does $Z$ [Kaw99]. Hence, if $\mu: X \rightarrow Z$ is any desingularization, and we have $f=g \mu$ with $K_{Z}$ locally free, then $f_{*} K_{X / \Delta}=g_{*} K_{Z / \Delta}$.

Proposition 2.8. Let $f: X \rightarrow \Delta$ be as at the beginning of this section, and suppose that $f^{\times}: X^{\times} \rightarrow \Delta^{\times}$admits a model $g: Z \rightarrow \Delta$ such that $Z$ is normal with $K_{Z}$ locally free and that $Z_{0}$ has only canonical singularities. Then $\alpha=\beta=0$.

Proof. Let $\mu: X^{\prime} \rightarrow Z$ be a normal crossings resolution and $X_{0}^{\prime}=\sum a_{i} E_{i}$, where $E_{0}=\widetilde{Z_{0}}$ is the strict transform of $Z_{0}$. Let $\eta \in K_{Z / \Delta}$ correspond to an element trivializing $g_{*} K_{Z / \Delta} \simeq$ 


\section{Eriksson, G. Freixas i Montplet and C. Mourougane}

$g_{*} \mu_{*} K_{X^{\prime} / \Delta}$. The divisor of $\mu^{*} \eta$ is then the divisor of the evaluation map for $X^{\prime}$; we denote it by $B=\sum\left(b_{i}-1\right) E_{i}$. Since $Z_{0}$ is normal and connected, it is integral, and since the evaluation map $g^{*} g_{*} K_{Z / \Delta} \rightarrow K_{Z / \Delta}$ cannot contain an entire fiber, it must be an isomorphism. It follows that $b_{0}=1$. By [Ste88, Theorem 2], if $Z_{0}$ has rational singularities, then for any exceptional $E_{i}$, we have $b_{i}-1 \geqslant a_{i}$. In characteristic zero, when the canonical sheaf is locally free, rational and canonical singularities are equivalent concepts, and since by adjunction $\left.K_{Z}\left(Z_{0}\right)\right|_{Z_{0}} \simeq K_{Z_{0}}$ is locally free, we infer that for any exceptional $E_{i}$, we have $b_{i} / a_{i} \geqslant 1+1 / a_{i}>1$. Moreover, for the non-exceptional component $\widetilde{Z_{0}}$, the ratio $b_{0} / a_{0}$ is 1 , so it follows immediately from Proposition 2.1 that $\alpha=\beta=0$.

Recall that $(X, x) \rightarrow(\Delta, 0)$, for $x \in X$, is an ordinary quadratic singularity if, locally on $X$, the map can be written as a germ of a holomorphic function $f:\left(\mathbb{C}^{n}, 0\right) \rightarrow(\mathbb{C}, 0)$ such that 0 is an isolated singularity of the level set $f=0$ and the Hessian of $f$ at 0 is invertible. Such singularities can all be diagonalized to the form $\sum z_{i}^{2}=0$. When $n \geqslant 2$, they are examples of canonical singularities, and $K_{X} \simeq \mathcal{O}_{X}$ because since the singular fiber is reduced and irreducible, the relative canonical divisor is zero, as seen at the end of Section 2.

Corollary 2.9. Suppose that $n \geqslant 2$ and $f: X \rightarrow \Delta$ has only ordinary quadratic singularities in $X_{0}$. Then $\alpha=\beta=0$.

Remark 2.10. Proposition 2.8 and Corollary 2.9 imply that if $X_{0}$ has only canonical singularities, or if $X$ is smooth and $X_{0}$ has only isolated ordinary quadratic singularities, then the $L^{2}$-metric is continuous.

\section{Degeneration of the Quillen metric}

\subsection{Background on Quillen metrics}

3.1.1 Grothendieck-Riemann-Roch in codimension 1. Let $f: X \rightarrow S$ be a smooth projective morphism of complex algebraic manifolds. We denote by $A_{*}(S)$ Fulton's intersection-theoretic Chow groups [Ful98]. Let $V$ be an algebraic vector bundle on $X$. The Grothendieck-RiemannRoch theorem with values in Chow groups is an identity of characteristic classes

$$
\operatorname{ch}\left(R f_{*} V\right)=f_{*}\left(\operatorname{ch}(V) \operatorname{Td}\left(T_{X / S}\right)\right) \in A_{*}(S)_{\mathbb{Q}} .
$$

The relation is also valid in de Rham cohomology. In this section, we focus on the "codimension 1 part" of the Grothendieck-Riemann-Roch formula. With values in Chow groups, this is written

$$
c_{1}\left(R f_{*} V\right)=f_{*}\left(\operatorname{ch}(V) \operatorname{Td}\left(T_{X / S}\right)\right)^{(1)} .
$$

The first Chern class of $R f_{*} V$ equals the first Chern class of the determinant of the cohomology $\operatorname{det} R f_{*} V$, also denoted by $\lambda(V)$. It can be defined by the theory of Knudsen-Mumford [KM76]. Contrary to the individual relative cohomology groups, it is compatible with base change.

3.1.2 Quillen metrics and the curvature formula. Suppose for simplicity that $X$ admits a Kähler metric, with Kähler form $\omega$, which we fix once and for all. If $V$ is equipped with a smooth Hermitian metric $h$ and $T_{X / S}$ with the restriction of the Kähler metric, then the Grothendieck-Riemann-Roch formula in codimension 1 can be lifted to the level of differential forms. This is achieved by means of Chern-Weil theory and the theory of the Quillen metric.

Let us briefly recall the definition of the Quillen metric. Let $s \in \mathrm{S}$, and consider the fiber 


\section{Metrics on Hodge Bundles}

of $\lambda(V)$ at $s$ :

$$
\lambda(V)_{s}=\bigotimes_{p} \operatorname{det} H^{p}\left(X_{s},\left.V\right|_{X_{s}}\right)^{(-1)^{p}} .
$$

By Hodge theory, and depending on the Hermitian metric $h$ and the Kähler form $\omega$ restricted to $X_{s}$, the cohomology groups $H^{p}\left(X_{s},\left.V\right|_{X_{s}}\right)$ carry $L^{2}$-type metrics (using the Dolbeault resolution and harmonic representatives). Hence, $\lambda(V)_{s}$ has an induced metric that we still call $L^{2}$-metric and that we write $h_{L^{2}, s}$. This family of metrics is in general not smooth in $s$, due to possible jumps in the dimensions of the cohomology. Let $T(s)$ be the holomorphic analytic torsion attached to $(V, h)$ and $\left(T_{X / S}, \omega\right)$ :

$$
T(s)=\sum_{p=0}^{n}(-1)^{p} p \log \operatorname{det} \Delta_{s}^{0, p} .
$$

Here, we denoted by $\Delta_{s}^{0, p}$ the $\bar{\partial}$-Laplacian acting on $A^{0, p}\left(\left.V\right|_{X_{s}}\right)$ (that is, $(0, p)$-forms on $X_{s}$ with values in $\left.V\right|_{X_{s}}$ ) and depending on the fixed Hermitian data. Also, $\operatorname{det} \Delta_{s}^{0, p}$ denotes the zetaregularized determinant of $\Delta_{s}^{0, p}$ (restricting to strictly positive eigenvalues). The Quillen metric on $\lambda(V)_{s}$ is defined by

$$
h_{\mathrm{Q}, s}=(\exp T(s)) h_{L^{2}, s} .
$$

This family of metrics is smooth in $s$. The resulting smooth metric on $\lambda(V)$ is called the Quillen metric, and we write $h_{Q}$ to refer to it. Observe that while the $L^{2}$-metric is defined using only harmonic forms (hence zero eigenforms for the Laplacians), the Quillen metric involves the whole spectrum of the Dolbeault Laplacians.

The curvature theorem of Bismut-Gillet-Soulé [BGS88a, BGS88b, BGS88c] is the equality of Chern-Weil differential forms on $S$

$$
c_{1}\left(\lambda(V), h_{\mathrm{Q}}\right)=f_{*}\left(\operatorname{ch}(V, h) \operatorname{Td}\left(T_{X / S}, \omega\right)\right)^{(1)} .
$$

By taking cohomology classes, one reobtains the Grothendieck-Riemann-Roch formula in de Rham cohomology.

3.1.3 The Quillen metric close to singular fibers. As a matter of motivation, we now review Yoshikawa's results [Yos07] on the degeneration of the Quillen metric in a slightly simplified form.

Let $f: X \rightarrow S$ be a generically smooth, flat and projective morphism of complex algebraic manifolds. Therefore, with respect to the previous setting, we allow for singular fibers. We assume that $S$ is 1-dimensional and $f$ has a unique singular fiber. Recall the Gauss map from the regular locus of $f$ to the space $\mathbb{P}(T X)$ of rank 1 quotients of $T X$ defined by

$$
\mu: X-\Sigma_{f} \rightarrow \mathbb{P}(T X), \quad x \mapsto T_{x} X / \operatorname{ker} d f_{x} .
$$

It is described in coordinates, through the isomorphism of $\mathbb{P}(T X)$ with the space $P\left(\Omega_{X} \otimes T S\right)=$ $\mathbb{P}\left(T X \otimes \Omega_{S}\right)$ of lines in $\Omega_{X} \otimes T S$, by

$$
\nu: X-\Sigma_{f} \rightarrow P\left(\Omega_{X} \otimes T S\right), \quad x \mapsto\left[\sum_{i=0}^{n} \frac{\partial s \circ f}{\partial z_{i}}(x) d z_{i} \otimes \frac{\partial}{\partial s}\right],
$$

where $\left(z_{i}\right)$ is a local coordinate system on $X$ and $s$ is a local coordinate on $S$. Consider the ideal sheaf $\mathcal{I}_{\Sigma_{f}}:=\left(\left((\partial s \circ f) / \partial z_{i}\right)(x)\right)$ on $X$ locally generated by the coefficients of $d f$. We resolve the singularities of $\mu$ and $\nu$ seen as meromorphic maps on $X$ by blowing up the ideal $\mathcal{I}_{\Sigma_{f}}$. Let 


\section{Eriksson, G. Freixas i Montplet and C. Mourougane}

$q: \widetilde{X} \rightarrow X$ be any desingularization of the blowup of this ideal and $E$ its exceptional divisor. We have a diagram
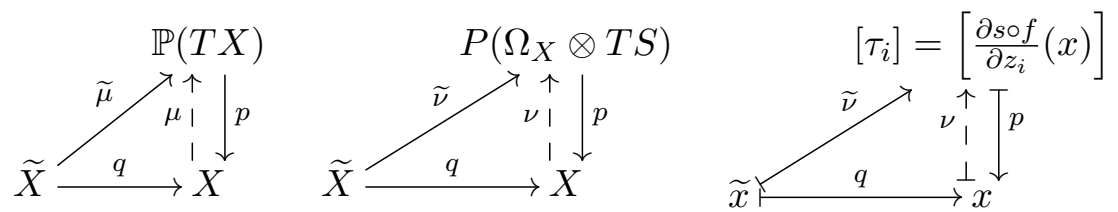

By construction, we see that $\widetilde{\nu}^{*} \mathcal{O}_{T X \otimes f^{*} \Omega_{S}}(1)=\mathcal{O}_{\widetilde{X}}(-E)$. Together with the isomorphism $\mathbb{P}(T X) \rightarrow \mathbb{P}\left(T X \otimes \Omega_{S}\right)$, this gives for the resolution $\widetilde{\mu}$ of $\mu$

$$
\tilde{\mu}^{*} \mathcal{O}_{T X}(1)=q^{*} f^{*} T S \otimes \mathcal{O}_{\widetilde{X}}(-E) .
$$

The tautological exact sequence on $\mathbb{P}(T X)$ hence pulls back on $\tilde{X}$ to

$$
0 \rightarrow \widetilde{\mu}^{*} U \rightarrow q^{*} T X \stackrel{q^{*} d f}{\longrightarrow} q^{*} f^{*} T S \otimes \mathcal{O}_{\widetilde{X}}(-E) \rightarrow 0,
$$

where $U$ denotes the tautological hyperplane subbundle. With these preliminaries at hand, we can now state the following result.

Theorem 3.1 (Yoshikawa [Yos07]). Fix a Kähler metric $h_{X}$ on $X$. Let $(V, h)$ be a holomorphic Hermitian vector bundle on $X$. On the smooth locus, equip the determinant line bundle $\lambda(V)$ with the corresponding Quillen metric.

(i) Let $\sigma$ be a local holomorphic frame for $\lambda(V)$ near the singular point $s=0$. Then

$$
\log \|\sigma\|_{\mathrm{Q}}^{2}=\left(\int_{E} \operatorname{Td} \tilde{\mu}^{*} U \frac{\operatorname{Td} \mathcal{O}_{\widetilde{X}}(-E)-1}{c_{1}\left(\mathcal{O}_{\tilde{X}}(-E)\right)} q^{*} \operatorname{ch}(V)\right) \log |s|^{2}+R(s) \quad \text { as } \quad s \rightarrow 0,
$$

where $R(s)$ is a continuous function of $s$.

(ii) The curvature current is given, in a neighborhood of $s=0$, by

$$
c_{1}\left(\lambda(V), h_{\mathrm{Q}}\right)=f_{*}\left(\operatorname{ch}(V, h) \operatorname{Td}\left(T_{X / S}, h_{X}\right)\right)^{(1,1)}-\left(\int_{E} \operatorname{Td} \widetilde{\mu}^{*} U \frac{\operatorname{Td} \mathcal{O}_{\widetilde{X}}(-E)-1}{c_{1}\left(\mathcal{O}_{\widetilde{X}}(-E)\right)} q^{*} \operatorname{ch}(V)\right) \delta_{0},
$$

where the first term on the right of the equality is $L_{\mathrm{loc}}^{p}(S)$ for some $p>1$ and $\delta_{0}$ is the Dirac current at 0.

(iii) Denote minus the coefficient of the logarithmic singularity by $\kappa$. Then the Quillen metric uniquely extends to a good Hermitian metric on the $\mathbb{Q}$-line bundle $\lambda(V) \otimes \mathcal{O}(-\kappa \cdot[0])$.

Remark 3.2. The third claim in the theorem is only implicitly stated in [Yos07]. In fact, it is proven that the potential of the curvature current of the Hermitian metric in part (iii) is of the form $\varphi(t)+\phi(t)$. Here $\varphi$ is smooth and $\phi$ is a finite sum of functions of the form $|s|^{2 r}(\log |s|)^{k} g(t)$, where $r \in \mathbb{Q} \cap(0,1]$, the exponent $k \geqslant 0$ is an integer and $g$ is smooth. This function and its derivatives satisfy the estimates in the definition of a good metric in the sense of Mumford [Mum77].

\subsection{The Nash blowup}

We proceed to develop an intersection-theoretic approach to Yoshikawa's theorem. Instead of the theory of the Gauss map and the resolution of the Jacobian ideal, we introduce the Grassmannian scheme and the Nash blowup. Throughout, we use the intersection theory of Fulton [Ful98]. The advantage of our construction is that our objects naturally exhibit a functorial behavior and that it allows for a better understanding of the topological term in Theorem 3.1 (cf. Definition 3.6). We recover and expand concrete computations of Yoshikawa. 


\section{Metrics on Hodge Bundles}

Let us say a word about the category where we place our arguments. We work in the category of schemes over $\mathbb{C}$, mostly to be in conformity with the literature. However, the relevant arguments should be applicable in the analytic category, using relative singular cohomology instead of bivariant Chow groups.

3.2.1 On the Jacobian ideal. Let $f: X \rightarrow S$ be a projective, flat, generically smooth morphism of integral Noetherian schemes over $\mathbb{C}$, of relative dimension $n$.

Define the Jacobian ideal $\mathcal{J} \operatorname{ac}(X / S)$ as the annihilator of $\Lambda^{n+1} \Omega_{X / S}$. Assume from now on that $X$ is locally a hypersurface in an $S$-smooth scheme $Y$ of dimension $n+1$. This is the case of hypersurfaces in $\mathbb{P}_{S}^{N}$, but also the case when $X$ and $S$ are smooth over $\mathbb{C}$ and $S$ is 1-dimensional (consider the graph of the morphism). Locally on $X$, we have an exact sequence

$$
0 \rightarrow \mathcal{I}_{X} / \mathcal{I}_{X}^{2} \stackrel{d}{\rightarrow} \Omega_{Y / S} \rightarrow \Omega_{X / S} \rightarrow 0
$$

where the ideal $\mathcal{I}_{X}$ of $X$ in $Y$ is generated by an element $F$. If one chooses (étale) local coordinates $y_{0}, \ldots, y_{n}$ on $Y$, then $\mathcal{J} \operatorname{ac}(X / S)$ is the $\mathcal{O}_{X}$-ideal generated by $\partial F / \partial y_{j}$ for $j=0, \ldots, n$. Observe that this is, by definition, the first Fitting ideal of $\Omega_{X / S}$. This local description shows that the Jacobian ideal is indeed the ideal defining the singular locus of the structure morphism $f$. For example, if $f: \mathcal{H} \rightarrow \check{\mathbb{P}}_{\mathbb{C}}^{N}$ is the tautological family of hyperplane sections in some smooth complex projective variety $X$, then the Jacobian ideal just corresponds to the scheme parametrizing singular sections.

3.2.2 On the Nash blowup. We still work locally on $X$. Locally, we denote by $Y$ a smooth $S$ scheme containing $X$ as a hypersurface. Let $\operatorname{Gr}_{n}\left(\Omega_{Y / S}\right)$ be the Grassmannian of rank $n$ quotients of $\Omega_{Y / S}$, and let $X \rightarrow \mathrm{Gr}_{n} \Omega_{Y / S}$ be the rational map defined by $x \mapsto\left(x, \Omega_{X / S, x}\right)$, called the Gauss map. The schematic closure $\widehat{X}$ of the image of this morphism is by definition the Nash blowup of $\Omega_{X / S}$ and has the universal property that an $S$-morphism $t: T \rightarrow \widehat{X}$ such that no component of $T$ has image contained in $V(\mathcal{J} \operatorname{ac}(X / S))$ corresponds to a surjection $\Omega_{X_{T} / T} \rightarrow \mathcal{E}$, where $\mathcal{E}$ is locally free of rank $n$ on $X_{T}$. Denote the obvious map by $\widehat{n}: \widehat{X} \rightarrow X$. As $\operatorname{Gr}_{n}\left(\Omega_{X / S}\right)$, understood as a Quot-scheme, is a closed subscheme of $\operatorname{Gr}_{n}\left(\Omega_{Y / S}\right)$, an equivalent definition, independent of the choice of the ambient space $Y$, is given by the closure of the $X / S$-smooth locus in $\operatorname{Gr}_{n}\left(\Omega_{X / S}\right)$. These constructions are summarized in the following diagram:

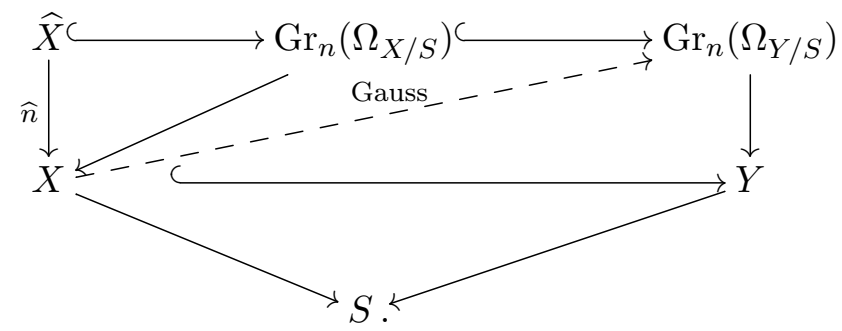

This gives another interpretation of the Gauss map, considered by Yoshikawa. Actually, suppose that $f: X \rightarrow S$ is a morphism of complex analytic manifolds, with $S$ of dimension 1 . Consider the graph $\Gamma_{f}: X \rightarrow S \times X$. Then the projection on $S$ from $Y=S \times X$ is smooth, and the map $X^{\text {sm }} \subseteq$ $\mathrm{Gr}_{n} \Omega_{X / S} \rightarrow \mathrm{Gr}_{n} \Omega_{X}$ from the $f$-smooth locus is given by $x \mapsto\left[\Omega_{X \times S / S, x}=\Omega_{X, x} \rightarrow \Omega_{X / S, x}\right]$. This is simply a dual version of the usual Gauss map. 


\section{Eriksson, G. Freixas i Montplet and C. Mourougane}

3.2.3 Comparison with the resolution of the Jacobian ideal. The Grassmannian construction, namely the Nash blowup, and the blowup of $X$ along the Jacobian ideal, actually coincide. This is useful in that both properties of blowups (structure of the exceptional divisor) and Grassmannians (existence of a universal locally free quotient and functoriality) can be simultaneously used.

Lemma 3.3 (see also [Pie79]). If $X$ is locally a hypersurface in an $S$-smooth scheme, then the blowup of $V(\mathcal{J a c}(X / S))$ in $X$ is the Nash blowup of $X$.

Proof. Denote by $b: X^{\prime} \rightarrow X$ the blowup of $X$ along $Z:=V(\mathcal{J} \operatorname{ac}(X / S))$ and by $\widehat{n}: \widehat{X} \rightarrow X$ the Nash blowup of $\Omega_{X / S}$. To construct a morphism from $X^{\prime}$ to $\widehat{X}$, we have to construct a rank $n$ locally free quotient of $\Omega_{X^{\prime} \times_{S} X / X^{\prime}}=b^{*} \Omega_{X / S}$. It is enough to show that the Gauss map locally extends to $X^{\prime}$, since local extensions are separated, hence unique. Locally, the ideal $\mathcal{I}_{X}$ of $X$ in some smooth $S$-scheme $Y$ is defined by an equation $F$ in $\mathcal{O}_{Y}$. Locally on $X^{\prime}$, the ideal $b^{*} \mathcal{J} \operatorname{ac}(X / S)$ is a free ideal $\mathcal{O}_{X^{\prime}}(-E)$ generated by an element $u$ that is not a zero divisor. The differential $b^{*} d F$ can then be written $u V$ for a uniquely determined nowhere-vanishing section $V$ in $b^{*} \Omega_{Y / S_{\mid X}}$. From the sequence (3.2) and the equality $V=$ " $b^{*} d F / u$ ", we infer that

$$
0 \rightarrow\left(b^{*} \mathcal{I}_{X} / \mathcal{I}_{X}^{2}\right) \otimes \mathcal{O}_{X^{\prime}}(E) \stackrel{d \otimes 1}{\longrightarrow} b^{*} \Omega_{Y / S_{\mid X}} \rightarrow b^{*} \Omega_{Y / S_{\mid X}} / V
$$

gives a locally well-defined locally free quotient $b^{*} \Omega_{X / S} \rightarrow b^{*} \Omega_{Y / S} / V$.

To construct a morphism from $\widehat{X}$ to $X^{\prime}$, by the universal property of blowing up, we have to show that the Jacobian ideal $\mathcal{J} \operatorname{ac}(X / S)$ becomes locally principal on $\widehat{X}$. Consider the following diagram on $\widehat{X}$, where the bottom line comes from the tautological sequence on $\operatorname{Gr}_{n}\left(\Omega_{Y / S}\right)$, the middle line comes from (3.2), the sheaf $M$ is the kernel of the rank $n$ quotient $\widehat{n}^{*} \Omega_{X / S} \rightarrow Q$ and $C$ is the fiber product of $\widehat{n}^{*} \Omega_{Y / S}$ and $M$ over $\widehat{n}^{*} \Omega_{X / S}$ :

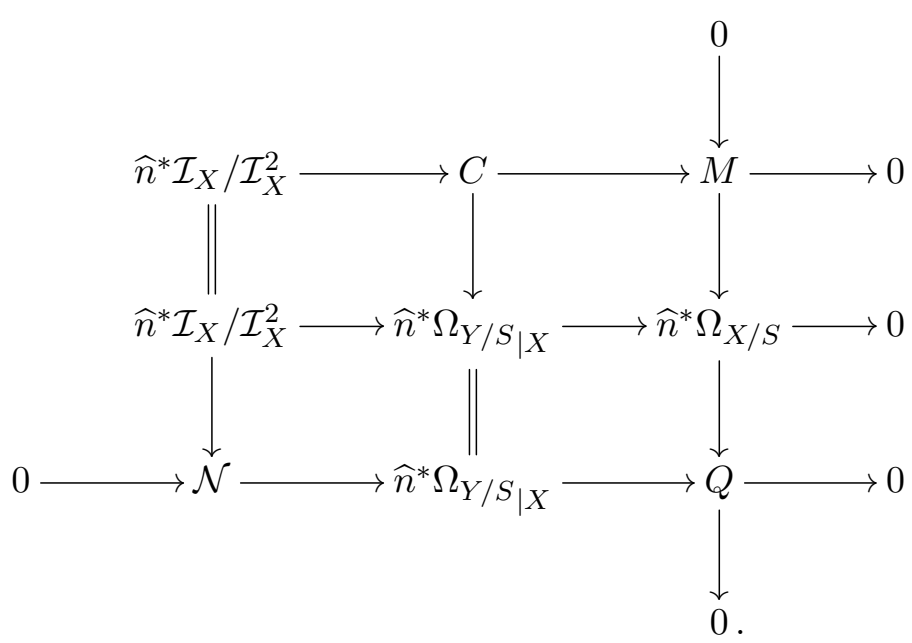

We infer an induced map $C \rightarrow \mathcal{N}$. As $C$ is a fiber product, a diagram chasing provides an inverse map $\mathcal{N} \rightarrow C$, so that $C$ is necessarily an invertible sheaf. The sheaf $Q$ being locally free, the Fitting ideal of $\widehat{n}^{*} \Omega_{X / S}$ is that of $M$, which is locally generated by the coefficient of the map $\widehat{n}^{*} \mathcal{I}_{X} / \mathcal{I}_{X}^{2} \rightarrow C$ between two invertible sheaves. By the functoriality of Fitting ideals, the pullback by $\widehat{n}$ of the Jacobian ideal is locally principal. The two constructed maps are inverse to each other over $X$, so that we can identify $b: X^{\prime} \rightarrow X$ and $\widehat{n}: \widehat{X} \rightarrow X$.

Thanks to the lemma, on the blowup $X^{\prime}$ of $X$ along the Jacobian ideal, there is a universal locally free quotient $b^{*} \Omega_{X / S} \rightarrow Q$ (coming from the Grassmannian interpretation). We now 


\section{Metrics on Hodge Bundles}

consider its kernel. Let $E$ be the exceptional divisor of the blowup $b: X^{\prime} \rightarrow X$, giving rise to the Cartesian diagram

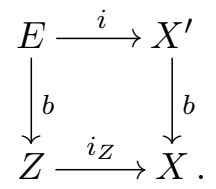

In the following lemma, $L^{i} f^{*}$ is the $i$ th left-derived inverse image under a morphism $f$. Recall that it is the sheaf defined by taking the $i$ th cohomology of the pull-back by $f$ of a locally free resolution. Note that the sheaf $\Omega_{X / S}$ admits locally free resolutions by the local hypersurface hypothesis. The lemma is to be compared with the dual of (3.1) restricted to $E$.

Lemma 3.4. Let $L_{E}$ be the kernel of the universal locally free quotient $b^{*} \Omega_{X / S} \rightarrow Q$. Then $L_{E}$ is a locally free sheaf of rank 1 on $E$. There is a canonical isomorphism

$$
L_{E} \simeq b^{*} L^{1} i_{Z}^{*} \Omega_{X / S} \otimes \mathcal{O}_{X^{\prime}}(E)_{\mid E}
$$

Furthermore, if $f: X \rightarrow S$ is a morphism of smooth algebraic varieties, then $L_{E} \simeq \mathcal{O}(E)_{\mid E}$.

Proof. That $L_{E}$ is supported on $E$ is immediate by construction. From the proof of the previous lemma, locally on $X$, there is a diagram of exact sequences

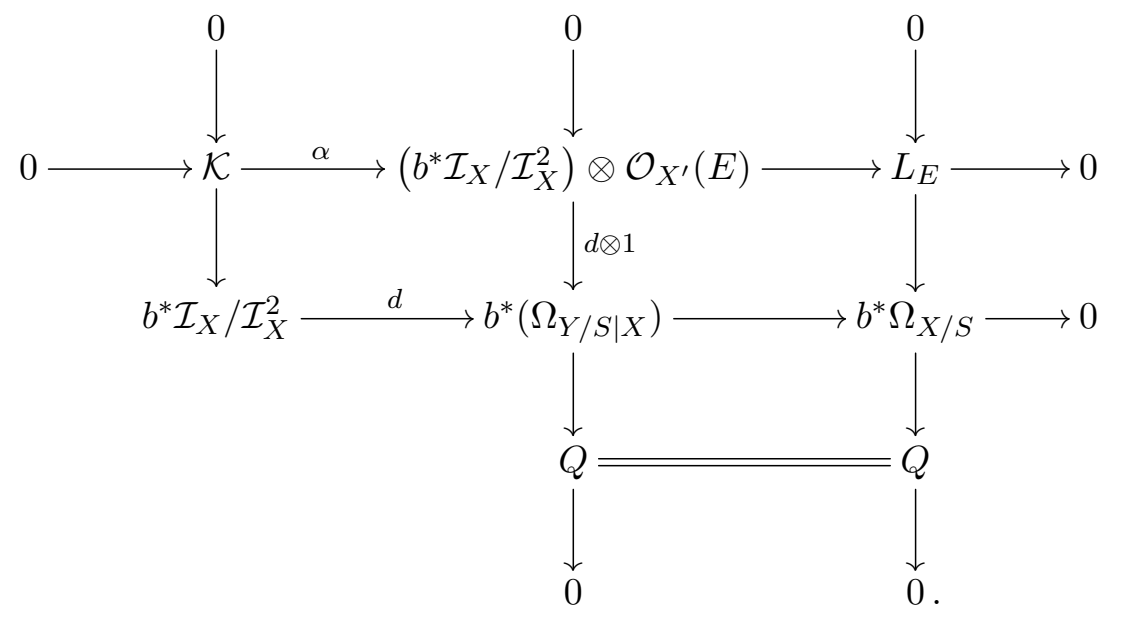

Because the differential $d: \mathcal{I}_{X} / \mathcal{I}_{X}^{2} \rightarrow \Omega_{Y / S}$ vanishes on $Z$, the induced map $b^{*} \mathcal{I}_{X} / \mathcal{I}_{X}^{2} \rightarrow b^{*} \Omega_{Y / S}$ vanishes on $E$ as well. Moreover, the morphism $d \otimes 1$ remains injective after restricting to $E$. It follows that $\alpha_{\mid E}$ vanishes identically, and hence there is an isomorphism

$$
L_{E} \simeq\left(b^{*} \mathcal{I}_{X} / \mathcal{I}_{X}^{2}\right)_{\mid E} \otimes \mathcal{O}_{X^{\prime}}(E)_{\mid E}
$$

This shows that $L_{E}$ is locally free of rank 1 . Now we claim that there is an isomorphism

$$
\left(b^{*} \mathcal{I}_{X} / \mathcal{I}_{X}^{2}\right)_{\mid E} \simeq b^{*} L^{1} i_{Z}^{*} \Omega_{X / S} .
$$

First of all, it is clear that $\left(b^{*} \mathcal{I}_{X} / \mathcal{I}_{X}^{2}\right)_{\mid E}=b^{*} i_{Z}^{*}\left(\mathcal{I}_{X} / \mathcal{I}_{X}^{2}\right)$. Second, from the long exact sequence associated with $i_{Z}^{*}$ applied to (3.2), we derive

$$
L^{1} i_{Z}^{*} \Omega_{X / S} \simeq i_{Z}^{*}\left(\mathcal{I}_{X} / \mathcal{I}_{X}^{2}\right) .
$$

The claim follows. Hence (3.4) and (3.5) give rise to an isomorphism as in the statement. One can check that it does not depend on the (local) choice of $Y$, so that it is a canonical isomorphism and globalizes. This completes the proof of the first claim. 


\section{Eriksson, G. Freixas i Montplet and C. Mourougane}

For the second assertion, it is enough to specialize the previous argument with $Y=X \times S$. In this case, it is immediate that $\mathcal{I}_{X} / \mathcal{I}_{X}^{2}=f^{*} \Omega_{S}$. Since $f$ is generically smooth and $S$ is 1 dimensional, the singular locus of $f$ in $S$ is 0 -dimensional. We thus see that

$$
L^{1} i_{Z}^{*} \Omega_{X / S} \simeq i_{Z}^{*}\left(\mathcal{I}_{X} / \mathcal{I}_{X}^{2}\right)=\left(f \circ i_{Z}\right)^{*}\left(\Omega_{S}\right)
$$

is a trivial line bundle.

\subsection{The Yoshikawa class}

3.3.1 Definition and properties of the Yoshikawa class. The previous notation and assumptions regarding the morphism $f: X \rightarrow S$ are still in force. In particular, $X$ is locally a hypersurface in a smooth $S$-scheme, $Z$ denotes the singular locus, the Nash blowup along $Z$ is $b: X^{\prime} \rightarrow X$ and $E$ is the exceptional divisor. We now digress on localized characteristic classes in the theory of Chow groups. This formalism, combined with the previous observations on Nash blowups, reveals useful to arrive at a conceptual explanation of the topological term in Yoshikawa's asymptotics. To be consistent with the literature on intersection theory and Chow groups (cf. Fulton's [Ful98], especially the relative setting of Chapter 20), from now on, we assume that $S$ is regular, for instance $\operatorname{Spec} R$ with $R$ a discrete valuation ring. Also, we will make extensive use of the theory of localized Chern classes. We refer the reader to [Ful98, Chapter 18.1] for the main construction of localized Chern classes of generically acyclic complexes, using the Grassmannian graph construction. We also cite [Abb00, Section 3] and [KS04, Section 2], which recast the main properties of the localized Chern classes of generically acyclic complexes in the form that will be used here.

Recall that a bivariant class $c \in A(X \rightarrow Y)$ is a rule that assigns, to every $Y$-scheme, say $Y^{\prime}$, a homomorphism

$$
c: A_{*}\left(Y^{\prime}\right) \longrightarrow A_{*}\left(X^{\prime}\right),
$$

where $X^{\prime}$ is the base change of $X$ to $Y^{\prime}$. This homomorphism is subject to several compatibilities (proper push-forward, flat pull-back and intersection product). We refer to [Ful98, Chapter 17] for the precise formulation of these.

Suppose that we are given a multiplicative characteristic class $T$, corresponding to a power series $T(x) \in 1+x \mathbb{Q}[x]$. Thus, with a vector bundle $\mathcal{E}$ on $X$ it associates homomorphisms on Chow groups $T(\mathcal{E}): A_{*}(X)_{\mathbb{Q}} \rightarrow A_{*}(X)_{\mathbb{Q}}$, and with a bounded complex of vector bundles $\mathcal{E}^{\bullet}$ it associates the homomorphism $\prod T\left(\mathcal{E}^{i}\right)^{(-1)^{i}}$, compatible with pull-backs. Let $b: X^{\prime} \rightarrow X$ be the Nash blowup of the morphism $f: X \rightarrow S$, with exceptional divisor $E$. On $X^{\prime}$, there is the universal locally free quotient $b^{*} \Omega_{X / S} \rightarrow Q$. Because $X$ is locally a hypersurface in a smooth $S$-scheme, this is quasi-isomorphic to a three-term complex of vector bundles. It is acyclic off the exceptional divisor $E$. Thus, following [Ful98, Chapter 18.1], there are localized bivariant Chern classes $c_{i}^{E}\left(b^{*} \Omega_{X / S} \rightarrow Q\right) \in A\left(E \rightarrow X^{\prime}\right)$, for $i>0$. Consequently, the class $T\left(b^{*} \Omega_{X / S} \rightarrow\right.$ $Q)-1=T\left(b^{*} \Omega_{X / S}\right) T(Q)^{-1}-1$ admits a refinement as a bivariant Chern class. Indeed, $T$ itself can be expressed as a power series in the Chern classes $c_{i}$, and the refinement to a bivariant class is obtained by replacing $c_{i}$ by $c_{i}^{E}$ in this power series representation. This refinement will be denoted by

$$
T^{E}\left(b^{*} \Omega_{X / S} \rightarrow Q\right) \in A\left(E \rightarrow X^{\prime}\right)_{\mathbb{Q}},
$$

or simply $T^{E}$ to simplify the notation. If $\left[X^{\prime}\right] \in A_{*}\left(X^{\prime}\right)$ is the cycle class of $X^{\prime}$, then $T^{E}$ sends $\left[X^{\prime}\right]$ into $A(E)_{\mathbb{Q}}$. The usual notation for this class is $T^{E} \cap\left[X^{\prime}\right]$. We will later be interested in the top-degree terms of such classes. 


\section{Metrics on Hodge Bundles}

The following lemma computes $T^{E} \cap\left[X^{\prime}\right]$ in terms of characteristic classes depending only on $\mathcal{O}(E)$.

Lemma 3.5. Assume that the base $S$ is 1-dimensional.

(i) As a bivariant class, $c_{i}\left(L^{1} i_{Z}^{*} \Omega_{X / S}\right)$ vanishes for $i \geqslant 1$. In particular, we have an equality of bivariant classes

$$
c_{1}\left(L_{E}\right)=c_{1}\left(\mathcal{O}(E)_{\mid E}\right) .
$$

(ii) The bivariant class $T^{E}$ satisfies the formula

$$
T^{E} \cap\left[X^{\prime}\right]=\left(\frac{T\left(\mathcal{O}(E)_{\mid E}\right)-1}{c_{1}\left(\mathcal{O}(E)_{\mid E}\right)} \cap[E]\right)
$$

in $A_{*}(E)_{\mathbb{Q}}$.

(iii) The bivariant class $T^{E}$ also satisfies the formula

$$
T\left(Q_{\mid E}\right)\left(T^{E} \cap\left[X^{\prime}\right]\right)=T\left(L i^{*} b^{*} \Omega_{X / S}\right)\left(\frac{1-T\left(\left.\mathcal{O}(E)\right|_{E}\right)^{-1}}{c_{1}\left(\left.\mathcal{O}(E)\right|_{E}\right)}\right) \cap[E] .
$$

Proof. For the first item, under our running assumptions on $X$ (locally hypersurface hypothesis, $f$ generically smooth and $S$ 1-dimensional and regular), the proof of [KS04, Lemma 5.1.3] can be adapted mutatis mutandis for $i=1$. For $i \geqslant 2$, the statement follows from the fact that $L^{1} i_{Z}^{*} \Omega_{X / S}$ is a line bundle. The equality $c_{1}\left(L_{E}\right)=c_{1}\left(\mathcal{O}(E)_{\mid E}\right)$ then follows from Lemma 3.4.

For the second claim, by a deformation to the normal cone argument with respect to the closed immersion $E \rightarrow X^{\prime}$, we can assume that $i: E \rightarrow X^{\prime}$ is the section of a projection $p: X^{\prime} \rightarrow E$. In this case, since $p_{*} i_{*}=\mathrm{Id}$, the direct image $i_{*}: A_{*}(E) \rightarrow A_{*}\left(X^{\prime}\right)$ is necessarily injective. Moreover, for any localized Chern class as in the statement,

$$
i_{*}\left(T^{E}\left(b^{*} \Omega_{X / S} \rightarrow Q\right) \cap\left[X^{\prime}\right]\right)=\left(T\left(b^{*} \Omega_{X / S} \rightarrow Q\right)-1\right) \cap\left[X^{\prime}\right] .
$$

On $X^{\prime}$, we have the tautological sequence

$$
0 \rightarrow L_{E} \rightarrow b^{*} \Omega_{X / S} \rightarrow Q \rightarrow 0 .
$$

Now, $L_{E}$ is a line bundle on $E$ by Lemma 3.4. Since $i: E \rightarrow X^{\prime}$ is a retraction, the line bundle $L=p^{*} L_{E}$ on $X^{\prime}$ extends $L_{E}$ and there is an exact sequence

$$
0 \rightarrow L(-E) \rightarrow L \rightarrow L_{E} \rightarrow 0 .
$$

We thus have a quasi-isomorphism of complexes

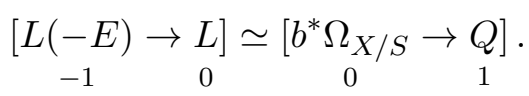

Consequently,

$$
\begin{aligned}
\left(T\left(b^{*} \Omega_{X / S} \rightarrow Q\right)-1\right) \cap\left[X^{\prime}\right] & =(T(L(-E) \rightarrow L)-1) \cap\left[X^{\prime}\right] \\
& =\left(T(L) T(L(-E))^{-1}-1\right) \cap\left[X^{\prime}\right] .
\end{aligned}
$$

The class $T(L) T(L(-E))^{-1}-1$ is naturally divisible by $c_{1}(\mathcal{O}(E))$. We can thus rewrite

$$
\left(T(L) T(L(-E))^{-1}-1\right) \cap\left[X^{\prime}\right]=\frac{T(L) T(L(-E))^{-1}-1}{c_{1}(\mathcal{O}(E))} \cap[E] .
$$

Finally, by Lemma 3.4, we also know that $L_{E}=\left.L^{1} i_{Z}^{*} \Omega_{X / S} \otimes \mathcal{O}(E)\right|_{E}$, and hence by the first item, we infer $c_{1}\left(L_{E}\right)=c_{1}\left(\mathcal{O}(E)_{\mid E}\right)$. Plugging this relation into (3.6), we arrive at the desired 


\section{Eriksson, G. Freixas i Montplet and C. Mourougane}

equality

$$
T^{E}\left(b^{*} \Omega_{X / S} \rightarrow Q\right) \cap\left[X^{\prime}\right]=\frac{T\left(\mathcal{O}(E)_{\mid E}\right)-1}{c_{1}\left(\mathcal{O}(E)_{\mid E}\right)} \cap[E] .
$$

The final claim follows the same lines (and notation) as the second, and the completely formal computations

$$
\begin{aligned}
i_{*}\left(T(Q) \cap T^{E} \cap\left[X^{\prime}\right]\right) & =T(Q)\left(T\left(b^{*} \Omega_{X / S}\right) T(Q)^{-1}-1\right) \cap\left[X^{\prime}\right] \\
& =T\left(b^{*} \Omega_{X / S}\right) \cap\left(1-T(Q) T\left(b^{*} \Omega_{X / S}\right)^{-1}\right) \cap\left[X^{\prime}\right] \\
& =T\left(b^{*} \Omega_{X / S}\right) \cap\left(1-T(L(-E)) T(L)^{-1}\right) \cap\left[X^{\prime}\right] .
\end{aligned}
$$

Recall that $\mathrm{Td}^{*}$ is the multiplicative characteristic class determined by $(-x) /\left(1-e^{-(-x)}\right)=$ $x /\left(e^{x}-1\right)$. We next define the Yoshikawa class, inspired by Theorem 3.1. We keep the assumptions of the introduction of this chapter.

Definition 3.6 (Yoshikawa class). Let $f: X \rightarrow S$ be a projective, flat, generically smooth morphism of integral Noetherian schemes over $\mathbb{C}$, of relative dimension $n$ with singular locus $i_{Z}: Z \hookrightarrow X$. Let $V$ be an algebraic vector bundle on $X$. Given a birational and proper morphism $\pi: \widetilde{X} \rightarrow X$ of integral schemes, with a surjection $\pi^{*} \Omega_{X / S} \rightarrow \mathcal{E}$ for some vector bundle $\mathcal{E}$ of rank $n$, define the Yoshikawa class as the cycle class

$$
\mathcal{Y}(X / S, V)=\operatorname{ch}\left(i_{Z}^{*} V\right) \cdot \pi_{*}\left(\operatorname{Td}^{*}\left(\mathcal{E}_{\mid D}\right) \operatorname{Td}^{* D}\left(\pi^{*} \Omega_{X / S} \rightarrow \mathcal{E}\right) \cap[\widetilde{X}]\right) \in A_{*}(Z)_{\mathbb{Q}},
$$

where $D:=\pi^{-1}(Z)$. For the trivial sheaf, we denote it by $\mathcal{Y}(X / S)$.

Proposition 3.7 (Independence). The Yoshikawa class is independent of the choice of a birational morphism $\pi: \widetilde{X} \rightarrow X$ and a surjection $\pi^{*} \Omega_{X / S} \rightarrow \mathcal{E}$.

Proof. The first assertion follows from the existence of the moduli of rank $d$ quotients of $\Omega_{X / S}$. Indeed, any datum as in the statement can be compared to the universal case on the Nash blowup: there exist a morphism to the Nash blowup $\varphi: \widetilde{X} \rightarrow X^{\prime}$ and a commutative diagram

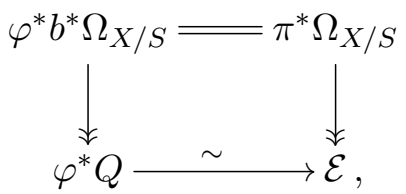

where the left-most vertical arrow is induced from the universal surjection on the Nash blowup. Moreover, we observe that

$$
L \varphi^{*} b^{*} \Omega_{X / S}=\varphi^{*} b^{*} \Omega_{X / S} .
$$

Indeed, since $X$ is Noetherian and is locally a hypersurface in an $S$-smooth scheme, $\Omega_{X / S}$ admits a two-term resolution by locally free sheaves $0 \rightarrow F_{1} \rightarrow F_{2} \rightarrow \Omega_{X / S} \rightarrow 0$. Notice that the pull-back $0 \rightarrow b^{*} F_{1} \rightarrow b^{*} F_{2} \rightarrow b^{*} \Omega_{X / S} \rightarrow 0$ is still exact, since the left-most map is generically injective on an integral scheme and hence globally injective. Repeating the argument with $\varphi$ establishes the relationship. We can then invoke the very construction of the localized Chern classes and the projection formula [Abb00,3.1, p. 31, especially $\left.\left(C_{1}\right)\right]$.

Yoshikawa's theorem works with a smooth desingularization of the Gauss map. The above proposition hence proves the following statement.

Corollary 3.8. Under the hypotheses of Theorem 3.1, the degree of $\mathcal{Y}(X / S, V)$ is the coefficient of the logarithmic singularity of the Quillen metric as in (1.1). 


\section{Metrics on Hodge BUndLes}

Remark 3.9. We expect that the hypothesis of smooth total space $X$ can be weakened with the same conclusion on the logarithmic singularity of the Quillen metric. This is one of the motivations of our treatment of the Yoshikawa class.

Proposition 3.10 (Functoriality). Suppose given a Cartesian diagram

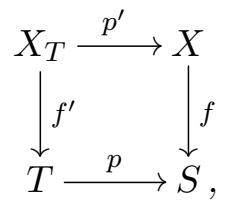

where $f^{\prime}$ is a generically smooth morphism of integral schemes and $p: T \rightarrow S$ is a locally complete intersection morphism. Then $p^{!} \mathcal{Y}(X / S, V)=\mathcal{Y}\left(X_{T} / T, p^{* *} V\right)$, where $p^{!}$denotes the refined Gysin morphism associated with $p$.

Proof. Let $Z^{\prime}$ be the Jacobian scheme of the morphism $f^{\prime}$. By the functoriality of Fitting ideals, the scheme $Z^{\prime}$ is the base change of $Z$ to $T$ and there is a canonical isomorphism $\left(X_{T}\right)^{\prime} \rightarrow\left(X^{\prime}\right)_{T}$ for the Nash blowups. In particular, it is legitimate to drop the parentheses in the notation. Factoring $T \rightarrow S$ as the composition of a smooth morphism and a regular closed immersion, we can treat each case separately. They are similar, but the smooth case is simpler, so we suppose henceforth that $T \rightarrow S$ is a regular closed immersion of constant codimension $d$. Now, consider the Cartesian diagrams

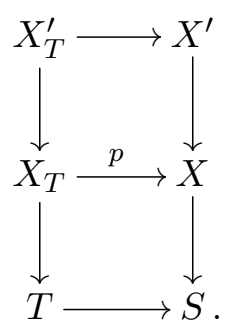

Any bivariant class $T^{E}$ with respect to $E \rightarrow X$ satisfies $p^{!}\left(T^{E} \cap[X]\right)=T^{E^{\prime}} \cap p^{!}\left[X^{\prime}\right]$ (see [Ful98, Section 17.1, axiom (C3)]), and clearly $p^{!}\left[X^{\prime}\right]=\left[X_{T}^{\prime}\right]$. Moreover, we have an induced Cartesian diagram

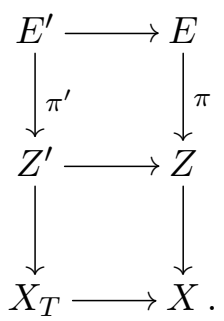

Then, as the refined Gysin maps commute with proper push-forward [Ful98, Theorem 6.2],

$$
\pi_{*}^{\prime}\left(T^{E^{\prime}} \cap\left[X_{T}^{\prime}\right]\right)=p^{!} \pi_{*}\left(T^{E} \cap[X]\right) .
$$

This implies the statement.

3.3.2 Computations of the Yoshikawa class. In the following proposition, we show that the Yoshikawa class can be written in terms of Segre classes (cf. [Ful98, Chapter 4]). In the particular case of isolated singularities and regular total space, the formula reduces to a classical topological invariant of those: the Milnor number. Recall that for a germ of an isolated hypersurface singularity $(Y, 0)$ determined by $f=0$ for a germ of a holomorphic map $f:\left(\mathbb{C}^{n+1}, 0\right) \rightarrow(\mathbb{C}, 0)$, 


\section{Eriksson, G. Freixas i Montplet and C. Mourougane}

the Milnor number is defined as

$$
\mu_{Y, 0}=\operatorname{dim}_{\mathbb{C}} \frac{\mathbb{C}\left\{z_{0}, \ldots, z_{n}\right\}}{\left(\partial f / \partial z_{0}, \ldots, \partial f / \partial z_{n}\right)} .
$$

In this setting, the Milnor number depends only on $(Y, 0)$ and not on the choice of smoothing function $f$.

The following results are a cohomological refinement of Yoshikawa's formulas [Yos98, Yos07].

Proposition 3.11. Let $f: X \rightarrow S$ be as before. Suppose that $S$ is 1-dimensional and $b: X^{\prime} \rightarrow X$ is the Nash blowup with exceptional divisor $E$.

(i) The Yoshikawa class fulfills the equality

$$
\mathcal{Y}(X / S)=\operatorname{Td}^{*}\left(i_{Z}^{*} \Omega_{X / S}\right) \cap \sum_{k=0}^{\infty} \frac{(-1)^{k+1}}{(k+2) !} s_{n-k}(Z),
$$

where $s_{n-k}(Z)=(-1)^{k} b_{*}\left(E^{k}\right) \in A_{n-k}(Z)$ is a Segre class.

(ii) If $Y$ is a smooth projective variety and $X \rightarrow S$ is a family of hypersurfaces in $Y \times S$, then

$$
\mathcal{Y}(X / S)=\operatorname{Td}^{*}\left(\Omega_{Y \mid Z}\right) \cap \sum_{k=0}^{\infty} \frac{(-1)^{k+1}}{(k+2) !} s_{n-k}(Z) .
$$

(iii) Suppose that $X \rightarrow S$ is the germ of a family over a disk $S=\operatorname{Spec} \mathbb{C}\{s\}$, locally a hypersurface in a smooth $S$-scheme, admitting only isolated singularities in the special fiber $X_{0}$; then

$$
\operatorname{deg} \mathcal{Y}(X / S)=\frac{(-1)^{n+1}}{(n+2) !} \sum_{x \in X_{0}}\left(\mu_{X, x}+\mu_{X_{0}, x}\right) .
$$

In particular, if $X$ is regular, then

$$
\operatorname{deg} \mathcal{Y}(X / S)=\frac{(-1)^{n+1}}{(n+2) !} \sum_{x \in X_{0}} \mu_{X_{0}, x} .
$$

Remark 3.12. In the third statement, the Milnor number $\mu_{X_{0}, x}$ is well defined since $\left(X_{0}, x\right)$ is locally a hypersurface in $\mathbb{C}^{n+1}$.

Proof. As in the proof of Proposition 3.7, one can show that

$$
L i^{*} b^{*} \Omega_{X / S} \simeq L i^{*} L b^{*} \Omega_{X / S} \simeq L b^{*} L i_{Z}^{*} \Omega_{X / S} .
$$

Moreover, by Lemma 3.5(a) and the observation, we have $L^{j} i_{Z}^{*} \Omega_{X / S}=0$ for $j \geqslant 2$ since there exists a local free resolution of length 2 of $\Omega_{X / S}$. The natural morphism $L i_{Z}^{*} \Omega_{X / S} \rightarrow i_{Z}^{*} \Omega_{X / S}$ hence has a kernel quasi-isomorphic to $L^{1} i_{Z}^{*} \Omega_{X / S}$, whose Chern classes are trivial by Lemma 3.5(a). We conclude that we have the relation $c_{j}\left(L i_{Z}^{*} \Omega_{X / S}\right)=c_{j}\left(i_{Z}^{*} \Omega_{X / S}\right)$ by the Whitney formula for Chern classes. With this understood, the first formula is a direct computation using the third claim in Lemma 3.5 and the projection formula.

For the second formula, by (3.2) applied with $Y \times S$ in place of $Y$, we see that $i_{Z}^{*} \Omega_{X / S}=\Omega_{Y \mid Z}$.

For the third property, we can suppose that $f: X \rightarrow S$ has an isolated singularity at a single closed point $x$ in the special fiber $X_{0}$. Furthermore, $i_{Z}^{*} \Omega_{X / S}$ is supported on a 0-dimensional space, and its Todd class is necessarily 1 . We then have, by the established formulas,

$$
\operatorname{deg} \mathcal{Y}(X / S)=\frac{(-1)^{n+1}}{(n+2) !} \operatorname{deg} s_{0}(Z)
$$




\section{Metrics on Hodge Bundles}

The rest of the argument closely follows [Tei74, Chapter II, Proposition 1.2]. Because $X \rightarrow S$ is Cohen-Macaulay and the singularity is isolated, the degree of the Segre class $s_{0}(Z)$ is computed by the colength of the Jacobian ideal [Ful98, Example 4.3.5(c)]. To compute the colength of the Jacobian ideal, we can reduce to the case of a germ $(X, 0) \rightarrow(\Delta, 0)$, where $(X, 0) \subseteq\left(\mathbb{C}^{n+1} \times \Delta, 0\right)$ is defined by $g=g\left(z_{0}, \ldots, z_{n}, s\right)=0$, with $\Delta$ the unit disk. Introduce the curve $\Gamma$ determined by the ideal $\left(\partial g / \partial z_{0}, \ldots, \partial g / \partial z_{n}\right)$. The colength of the Jacobian ideal is then given by

$$
\operatorname{dim}_{\mathbb{C}} \frac{\mathbb{C}\left\{z_{0}, \ldots, z_{n}, s\right\}}{\left(g, \partial g / \partial z_{0}, \ldots, \partial g / \partial z_{n}\right)}=g \cdot \Gamma,
$$

where the right-hand side denotes the intersection number of $\{g=0\}$ and $\Gamma$. Denote the normalization map by $\pi: \widetilde{\Gamma} \rightarrow \Gamma$. By the projection formula, we have $g \cdot \Gamma=\pi^{*} g \cdot \widetilde{\Gamma}$. For its computation, for $x_{i} \in \pi^{-1}(0)$, choose a local coordinate $s_{i}$ and denote the canonical discrete valuation at $x_{i}$ by $v_{i}$. The intersection number is then given by

$$
\pi^{*} g \cdot \widetilde{\Gamma}=\sum v_{i}\left(\pi^{*} g\right) .
$$

Notice that for a function $h$ vanishing on $x_{i}$, we have $v_{i}\left(\partial h / \partial s_{i}\right)=v_{i}(h)-1$. By the chain rule, we find that

$$
\frac{\partial \pi^{*} g}{\partial s_{i}}=\pi^{*}\left(\frac{\partial g}{\partial s}\right) \frac{\partial \pi^{*} s}{\partial s_{i}}+\sum \pi^{*}\left(\frac{\partial g}{\partial z_{i}}\right) \frac{\partial \pi^{*} z_{i}}{\partial s_{i}}=\pi^{*}\left(\frac{\partial g}{\partial s}\right) \frac{\partial \pi^{*} s}{\partial s_{i}} .
$$

We conclude that

$$
\begin{aligned}
v_{i}\left(\pi^{*} g\right)=v_{i}\left(\frac{\partial \pi^{*} g}{\partial s_{i}}\right)+1 & =v_{i}\left(\pi^{*}\left(\frac{\partial g}{\partial s}\right)\right)+v_{i}\left(\frac{\partial \pi^{*} s}{\partial s_{i}}\right)+1 \\
& =v_{i}\left(\pi^{*}\left(\frac{\partial g}{\partial s}\right)\right)+v_{i}\left(\pi^{*} s\right),
\end{aligned}
$$

from which we find $g \cdot \Gamma=\partial g / \partial s \cdot \Gamma+s \cdot \Gamma$. These are Milnor numbers as defined in (3.7), which proves the statement. In the special case where $X$ is moreover regular, $\mu_{X, x}=0$ for every point $x \in X$.

The following lemma will be useful in some computations with the Yoshikawa class. As an example is of use, we refer to Theorems 3.14 and 3.16 below.

Lemma 3.13. Let $f: X \rightarrow S$ be a germ of a fibration over the unit disk, with regular total space $X$. Then

$$
\operatorname{deg} c_{n}\left(Q_{\mid E}\right)=\operatorname{deg} c_{n+1}^{Z}\left(\Omega_{X / S}\right) \cap[X]=(-1)^{n}\left(\chi\left(X_{\infty}\right)-\chi\left(X_{0}\right)\right),
$$

where $Z \subset X$ is the singular locus of $f, X_{\infty}$ is a general fiber and $\chi$ is the topological Euler characteristic.

Proof. For the equality

$$
\operatorname{deg} c_{n+1}^{Z}\left(\Omega_{X / S}\right) \cap[X]=(-1)^{n}\left(\chi\left(X_{\infty}\right)-\chi\left(X_{0}\right)\right),
$$

we observe that

$$
\operatorname{deg} c_{n+1}^{Z}\left(\Omega_{X / S}\right) \cap[X]=\operatorname{deg} c_{n+1}^{X_{0}}\left(\Omega_{X / S}\right) \cap[X]
$$

and then we refer to [Ful98, Example 14.1.5].

For the first equality, we recall from Lemma 3.4 the tautological exact sequence on the Nash blowup $X^{\prime}$

$$
0 \rightarrow L_{E} \rightarrow b^{*} \Omega_{X / S} \rightarrow Q \rightarrow 0
$$




\section{Eriksson, G. Freixas i Montplet and C. Mourougane}

By the Whitney formula for localized Chern classes [Abb00, Proposition 3.1(b)] and the vanishing property in Lemma 3.5(a), we have

$$
c_{n+1}^{E}\left(b^{*} \Omega_{X / S}\right) \cap\left[X^{\prime}\right]=c_{n}\left(Q_{\mid E}\right)\left(c_{1}^{E}\left(L_{E}\right) \cap\left[X^{\prime}\right]\right)=c_{n}\left(Q_{\mid E}\right) \cap[E] .
$$

On the other hand, we apply the projection formula of localized Chern classes with respect to proper morphisms $\left[\mathrm{Abb} 00,3.1,\left(C_{1}\right)\right.$, p. 31], which implies

$$
\operatorname{deg} c_{n+1}^{E}\left(b^{*} \Omega_{X / S}\right) \cap\left[X^{\prime}\right]=\operatorname{deg} c_{n+1}^{Z}\left(\Omega_{X / S}\right) \cap[X] .
$$

We complete the proof by combining the last two equalities.

3.3.3 The Yoshikawa class for families of hypersurfaces. Recall that the discriminant or dual variety of a smooth variety $Y \subseteq \mathbb{P}^{N}$ is a variety $\Delta_{Y} \subseteq \check{\mathbb{P}}^{N}$ parametrizing the hyperplanes $H \in \check{\mathbb{P}}^{N}$ such that $Y \cap H$ is singular. Here $Y \cap H$ is regarded as a scheme. In many interesting cases, $\Delta_{Y}$ is a hypersurface. Let us mention the case of the $d$-Veronese embedding $\mathbb{P}^{n} \subseteq \mathbb{P}^{N}$. In this case, $\Delta_{Y}$ parametrizes singular hypersurfaces of degree $d$ in $\mathbb{P}^{n}$.

We denote the universal family of hyperplane sections of $Y$ by $F: \mathcal{H} \rightarrow \check{\mathbb{P}}^{N}$. The $F$-singular locus can be described as the projective bundle $\mathbb{P}\left(N_{Y / \mathbb{P}^{N}}\right)$ over $Y$, where $N$ denotes the normal bundle of $Y \subseteq \mathbb{P}^{N}$. Indeed, a singular point in a hyperplane section is nothing but a hyperplane $H$ and a point $y \in Y \cap H$ such that $T_{y} H \subseteq T_{y} \mathbb{P}^{n}$ contains $T_{y} Y$, so that $H$ corresponds to a vector in $\mathbb{P}\left(N_{Y / \mathbb{P}^{N}, y}\right)$, the projectivized normal bundle of $Y \subseteq \mathbb{P}^{n}$ at $y$. Hence the $F$-singular locus is just the projectivized normal bundle of $Y \subseteq \mathbb{P}^{N}$ [GKZ08, Chapter 1, Section 3A, p. 27]. In particular, $\Delta_{Y}$, being the image of $\mathbb{P}\left(N_{Y / \mathbb{P}^{N}}\right)$ in $\check{\mathbb{P}}^{N}$, is irreducible.

Theorem 3.14. Suppose that $f: X \rightarrow S$ is a family of hyperplane sections of a smooth complex projective variety $Y \subseteq \mathbb{P}^{N}$ of dimension $n+1$, over a regular base $S$. Let $Z$ be the singular scheme of $f$. Then the codimension $n+1$ component of $\mathcal{Y}(X / S)$ is given by

$$
\mathcal{Y}(X / S)^{(n+1)}=\frac{(-1)^{n+1}}{(n+2) !} c_{n+1}^{Z}\left(\Omega_{X / S}\right) \cap[X] .
$$

Consequently,

$$
\operatorname{deg} \mathcal{Y}(X / S)=\frac{(-1)^{n+1}}{(n+2) !} \int_{X_{0}} c_{n+1}^{X_{0}}\left(\Omega_{X / S}\right) \cap[X]
$$

Remark 3.15. In the context of Theorem 3.14, when $X$ is regular and $S$ is 1-dimensional, one can see that $f$ has at most isolated singularities. Then, according to the theorem and Lemma 3.13, the degree of the Yoshikawa class is given by the change of Euler characteristics or, equivalently, the vanishing cycles. This is compatible with Proposition 3.11(c) since the sum of the Milnor numbers equals the number of vanishing cycles.

Proof. For the first point, by Proposition 3.10 and the analogous functoriality for $c_{n+1}^{Z}\left(\Omega_{X / S}\right) \cap$ $[X]$, it is enough to prove that

$$
\mathcal{Y}(X / S)^{(n+1)}=\frac{(-1)^{n+1}}{(n+2) !} c_{n+1}^{Z}\left(\Omega_{X / S}\right) \cap[X]
$$

when $X \rightarrow S$ is the universal situation $\mathcal{H} \rightarrow \check{\mathbb{P}}^{N}$ with $Z=\mathbb{P}(N)$.

We start by proving that

$$
[Z]=c_{n+1}^{Z}\left(\Omega_{\mathcal{H} / \check{\mathbb{P}}^{N}}\right) \cap[\mathcal{H}]
$$




\section{Metrics on Hodge Bundles}

and later we will relate the Yoshikawa class to $[\mathbb{P}(N)]$. Consider the resolution

$$
\mathcal{O}(-\mathcal{H})_{\mid \mathcal{H}} \rightarrow \Omega_{Y \times \check{\mathbb{P}}^{N} / \check{\mathbb{P}}^{N} \mid \mathcal{H}} \rightarrow \Omega_{\mathcal{H} / \check{\mathbb{P}}^{N}} \rightarrow 0 .
$$

It determines a section $\sigma$ of $\Omega_{Y \times \check{\mathbb{P}}^{N} / \check{\mathbb{P}}^{N}}(\mathcal{H})_{\mid \mathcal{H}}$ whose schematic zero locus is $Z$. This is of maximal codimension $n+1$ in $\mathcal{H}$; hence by [Ful98, Proposition 14.1(c)], the corresponding localized Chern class is given by $[Z]$. All in all, we conclude that we have

$$
[Z]=c_{n+1}^{Z}\left(\Omega_{\mathcal{H} / \check{\mathbb{P}}^{N}} \otimes \mathcal{O}(\mathcal{H})_{\mid \mathcal{H}}\right)=c_{n+1}^{Z}\left(\Omega_{\mathcal{H} / \check{\mathbb{P}}^{N}}\right),
$$

where the last equality is easily checked from the very construction of the localized Chern classes through the Grassmannian graph construction (see [Abb00, Section 3] and use that $\mathcal{O}(\mathcal{H})_{\mid \mathcal{H}}$ is invertible; hence tensoring by it induces an isomorphism on Grassmannians and does not alter the construction in $[\mathrm{Abb} 00])$.

Now, we compute the $(N-1)$-dimensional component of the Yoshikawa class in the universal situation. First, we observe that the codimension $n+1$ component $\mathcal{Y}\left(\mathcal{H} / \check{\mathbb{P}}^{N}\right)$ is concentrated on the $(N-1)$-dimensional irreducible subscheme $Z$, and hence is a multiple thereof:

$$
\mathcal{Y}\left(\mathcal{H} / \check{\mathbb{P}}^{N}\right)^{(n+1)}=m[Z]
$$

for some rational number $m$. Second, we determine the coefficient $m$ by "evaluating" on a point. For this, let $b: \mathcal{H}^{\prime} \rightarrow \mathcal{H}$ be the Nash blowup. The induced map $E \rightarrow Z$ has the structure of a projective bundle of rank $n$. As in the proof of Lemma 3.5, write the Yoshikawa class as

$$
b_{*}\left(\operatorname{Td}^{*}\left(L i^{*} b^{*} \Omega_{\mathcal{H} / \check{\mathbb{P}}^{N}}\right) \cap\left(\frac{1-\mathrm{Td}^{*}\left(L_{E}(-E)\right) \mathrm{Td}^{*}\left(L_{E}\right)^{-1}}{c_{1}\left(\mathcal{O}(E)_{\mid E}\right)}\right) \cap[E]\right),
$$

where $i$ is the closed immersion of $E$ into $\mathcal{H}^{\prime}$. Let $k: p \rightarrow Z$ be any (closed) point of $Z$, necessarily a closed regular immersion of codimension $N-1$. Then we have a Cartesian diagram

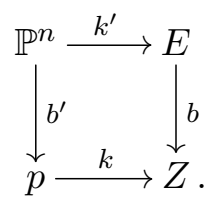

Then as $k^{*}[Z]=[p]$, it is enough to compute $k^{*} \mathcal{Y}\left(\mathcal{H} / \check{\mathbb{P}}^{N}\right)$. We obviously have that

$$
k^{\prime *} b^{*} L^{1} i_{Z}{ }^{*} \Omega_{\mathcal{H} / \check{\mathbb{P}}^{N}}=b^{\prime *} k^{*} L^{1} i_{Z}{ }^{*} \Omega_{\mathcal{H} / \check{\mathbb{P}}^{N}}
$$

is a trivial line bundle over a point. Therefore, by Lemma 3.4, we find $k^{\prime *} L_{E}=\mathcal{O}(E)_{\mathbb{P}^{n}}=\mathcal{O}(-1)$. Furthermore, $b_{*} k^{*}=b^{\prime}{ }_{*} k^{\prime *}$ and we conclude that the pull-back of the Yoshikawa class is given by $\int_{\mathbb{P}^{n}}\left(1-\operatorname{Td}^{*}(\mathcal{O}(-1))^{-1}\right) c_{1}(\mathcal{O}(-1))^{-1}$. This further simplifies to

$$
m=\operatorname{deg} k^{*} \mathcal{Y}\left(\mathcal{H} / \check{\mathbb{P}}^{N}\right)=\frac{(-1)^{n+1} c_{1}(\mathcal{O}(1))^{n}}{(n+2) !}=\frac{(-1)^{n+1}}{(n+2) !} .
$$

The consequence

$$
\operatorname{deg} \mathcal{Y}(X / S)=\frac{(-1)^{n+1}}{(n+2) !} \int_{X_{0}} c_{n+1}^{X_{0}}\left(\Omega_{X / S}\right) \cap[X]
$$

follows by the properties of localized Chern classes and the fact that $c_{n+1}^{X_{0}}\left(\Omega_{X / S}\right)$ is supported on the singular locus $Z$.

3.3.4 The Yoshikawa class for Kulikov families of surfaces. We now look at a germ of a Kulikov family over a disk, $f: X \rightarrow S$. We assume that $X$ is regular, that $f$ has relative dimension 2 


\section{Eriksson, G. Freixas i Montplet and C. Mourougane}

and a unique singular fiber over 0 , and finally that the relative canonical sheaf $K_{X}$ is trivial. Observe that we do not require the general fiber to be a K3 surface; hence we also allow it to be an abelian surface.

Theorem 3.16. The Yoshikawa class of a Kulikov family as above satisfies

$$
\operatorname{deg} \mathcal{Y}(X / S)=\frac{-1}{24}\left[\chi\left(X_{\infty}\right)-\chi\left(X_{0}\right)\right]
$$

Proof. Let $b: X^{\prime} \rightarrow X$ be the Nash blowup, with universal quotient bundle $Q$ and exceptional divisor $E$. A direct computation using Lemma 3.5(b) shows that the degree is given by

$$
\operatorname{deg} \mathcal{Y}(X / S)=\int_{E} \frac{-c_{1}(Q) c_{1}(\mathcal{O}(E))-c_{1}(Q)^{2}-c_{2}(Q)}{24} .
$$

Recall the exact sequence

$$
0 \rightarrow L_{E} \rightarrow b^{*} \Omega_{X / S} \rightarrow Q \rightarrow 0
$$

which together with Lemma 3.5(a) implies

$$
c_{1}\left(\left.b^{*} K_{X / S}\right|_{E}\right) \cap\left[X^{\prime}\right]=c_{1}\left(\left.\mathcal{O}(E)\right|_{E}\right) \cap\left[X^{\prime}\right]+c_{1}\left(\left.Q\right|_{E}\right) \cap\left[X^{\prime}\right] .
$$

But by the Kulikov assumption, $K_{X / S}$ is trivial, and therefore

$$
c_{1}\left(\left.\mathcal{O}(E)\right|_{E}\right) \cap\left[X^{\prime}\right]=-c_{1}\left(\left.Q\right|_{E}\right) \cap\left[X^{\prime}\right] .
$$

Plugging this relation into (3.8), we find

$$
\operatorname{deg} \mathcal{Y}(X / S)=-\int_{E} \frac{c_{2}(Q)}{24} .
$$

We conclude by Lemma 3.13.

\section{Degeneration of the $\mathrm{BCOV}$ metric}

In this section, we will consider families of Calabi-Yau varieties and their BCOV line bundles. More precisely, we will study the BCOV metric introduced by [FLY08] and its asymptotic behavior under degeneration. We will use the results in the preceding sections to show that the singularity is governed by topological invariants, especially vanishing cycles in the case of Kulikov families.

For the rest of this section, let $f: X \rightarrow S$ be a generically smooth flat projective morphism of complex algebraic manifolds with connected fibers and $\operatorname{dim} S=1$. We suppose that the nonsingular fibers are $n$-dimensional Calabi-Yau varieties, in the sense that their canonical bundles are trivial. We suppose that $X$ has a fixed Kähler metric $h_{X}$.

\subsection{The BCOV line bundle and metric}

We define the BCOV line bundle. First, assume that $f$ is smooth. Then, put

$$
\begin{aligned}
\lambda_{\mathrm{BCOV}}\left(\Omega_{X / S}^{\bullet}\right) & :=\lambda\left(\bigoplus_{0 \leqslant p \leqslant n}(-1)^{p} p \Omega_{X / S}^{p}\right)=\bigotimes_{0 \leqslant p \leqslant n} \lambda\left(\Omega_{X / S}^{p}\right)^{(-1)^{p} p} \\
& =\bigotimes_{0 \leqslant p, q \leqslant n}\left(\operatorname{det} R^{q} f_{*}\left(\Omega_{X / S}^{p}\right)\right)^{(-1)^{p+q} p} .
\end{aligned}
$$

In general, the sheaves $\Omega_{X / S}^{p}$ are only coherent sheaves on $X$ and not locally free. To extend the BCOV line bundle from the smooth locus to the whole base $S$, it is useful to introduce the so- 


\section{Metrics on Hodge Bundles}

called Kähler resolution of $\Omega_{X / S}^{p}$, involving the locally free sheaves $\Omega_{X}^{p}$ and $f^{*} \Omega_{S}^{\otimes q}$. Equivalently, we apply the left-derived functor $L \Lambda^{p}$ to $\Omega_{X / S}$. This is achieved by simply applying the exterior power functors to the exact sequence (2.1) defining the relative cotangent sheaf. For each $0 \leqslant$ $p \leqslant n$, we obtain a complex

$$
\widetilde{\Omega_{X / S}^{p}}:\left(f^{*} \Omega_{S}\right)^{\otimes p} \rightarrow\left(f^{*} \Omega_{S}\right)^{\otimes p-1} \otimes \Omega_{X} \rightarrow \cdots \rightarrow\left(f^{*} \Omega_{S}\right) \otimes \Omega_{X}^{p-1} \rightarrow \Omega_{X}^{p} .
$$

The Kähler extension $\lambda_{\mathrm{BCOV}}\left(\widetilde{\Omega_{X / S}^{\bullet}}\right)$ of the BCOV line bundle on the smooth locus is then defined to be

$$
\lambda_{\mathrm{BCOV}}\left(\widetilde{\Omega_{X / S}^{\bullet}}\right)=\lambda\left(\underset{0 \leqslant p \leqslant n}{\bigoplus}(-1)^{p} p \widetilde{\Omega_{X / S}^{p}}\right)=\bigotimes_{p=0}^{n} \bigotimes_{j=0}^{p}\left(f^{*} \Omega_{S}\right)^{(-1)^{p+j} p j} \lambda\left(\Omega_{X}^{p-j}\right)^{(-1)^{p+j} p} .
$$

For smooth $f$, and depending on the Kähler metric $h_{X}$, the BCOV line bundle carries a combination of Quillen metrics. We now introduce the BCOV metric, following [FLY08, Definition 4.1], but phrased differently.

Definition 4.1. (i) The function $A(X / S) \in \mathcal{C}^{\infty}(S)$ is locally given by the formula

$$
A(X / S)=\left\|\eta_{X / S}\right\|_{L^{2}}^{\chi\left(X_{\infty}\right) / 6} \exp \left\{\frac{(-1)^{n+1}}{12} f_{*}\left(\log \left(\frac{\left\|\eta_{X}\right\|^{2}}{\|d f\|^{2}}\right) c_{n}\left(\Omega_{X / S}, h_{X}\right)\right)\right\} .
$$

Here, $\eta_{X}$ is a nowhere-vanishing global section of $K_{X}$ (which exists locally relative to the base) and $\eta_{X / S}$ is the Gelfand-Leray residue form of $\eta_{X}$ with respect to $f$, namely the section of $f_{*}\left(K_{X / S}\right)$ determined by $\eta_{X}=\eta_{X / S} \wedge f^{*}(d s)$, for some local coordinate $s$ on $S$.

(ii) The BCOV metric on $\lambda_{\mathrm{BCOV}}\left(\Omega_{X / S}^{\bullet}\right)$ is

$$
h_{\mathrm{BCOV}}=A(X / S) h_{\mathrm{Q}},
$$

where $h_{\mathrm{Q}}$ is the Quillen metric depending on $h_{X}$.

The following statement describes the singular behavior of the BCOV metric when the morphism $f: X \rightarrow S$ is only supposed to be generically smooth.

Proposition 4.2. Let $f: X \rightarrow S$ be a generically smooth family of Calabi-Yau varieties of dimension $n$. Assume that there is at most one singular fiber of equation $s=0$. We denote by $\alpha$ and $\beta$ the coefficients encoding the asymptotics of the $L^{2}$-metric in Proposition 2.3.

(i) Choose a local holomorphic frame $\widetilde{\sigma}$ for the Kähler extension $\lambda_{\mathrm{BCOV}}\left(\widetilde{\Omega_{X / S}^{\bullet}}\right)$. Set

$$
\begin{aligned}
\alpha_{\mathrm{BCOV}}= & \sum_{p=0}^{n} \sum_{j=0}^{p} \int_{E}\left(p(-1)^{j} \operatorname{Td}^{*} Q \frac{\operatorname{Td}^{*} \mathcal{O}_{\widetilde{X}}(E)-e^{(p-j) c_{1}\left(\mathcal{O}_{\tilde{X}}(E)\right)}}{c_{1}\left(\mathcal{O}_{\widetilde{X}}(E)\right)} q^{*} \operatorname{ch}\left(\Omega_{X}^{j}\right)\right) \\
& +\frac{1}{12}\left(-\left(\chi\left(X_{\infty}\right)-\chi\left(X_{0}\right)\right)+\alpha \chi\left(X_{\infty}\right)+(-1)^{n} \int_{b^{*} B} c_{n}(Q)\right),
\end{aligned}
$$

where $X^{\prime} \stackrel{b}{\rightarrow} X$ is the Nash blowup of $f, E$ its exceptional divisor, $Q$ the tautological quotient vector bundle on $X^{\prime}$ and $B$ the divisor of the evaluation map (2.2). Then the asymptotic of the BCOV norm of $\widetilde{\sigma}$ as $s \rightarrow 0$ is

$$
-\log \|\widetilde{\sigma}\|_{\mathrm{BCOV}}^{2}=\alpha_{\mathrm{BCOV}} \log |s|^{2}-\left.\frac{\chi\left(X_{\infty}\right)}{12} \beta \log |\log | s\right|^{2} \mid+ \text { continuous . }
$$




\section{Eriksson, G. Freixas i Montplet and C. Mourougane}

(ii) The BCOV metric uniquely extends to a good metric (in the sense of Mumford) on the $\mathbb{Q}$-line bundle $\lambda_{\mathrm{BCOV}}\left(\widetilde{\Omega_{X / S}^{\bullet}}\right) \otimes \mathcal{O}\left(-\alpha_{\mathrm{BCOV}}[0]\right)$. It has an $L^{p}(p>1)$ potential

$$
-\left.\left(\chi\left(X_{\infty}\right) / 12\right) \beta \log |\log | s\right|^{2} \mid+ \text { continuous . }
$$

(iii) Suppose that $f: X \rightarrow S$ is smooth and is the restriction of a Kuranishi family under a classifying map $\iota$. Then the curvature form of the BCOV metric agrees with the pull-back of the Weil-Petersson form

$$
c_{1}\left(\lambda_{\mathrm{BCOV}}\left(\Omega_{X / S}^{\bullet}\right), h_{\mathrm{BCOV}}\right)=\frac{\chi\left(X_{\infty}\right)}{12} \iota^{*} \omega_{\mathrm{WP}} .
$$

Proof. The first equality is the conjunction of the asymptotic formulas of the Quillen metric and computations and asymptotics of the term $A(X / S)$. The Quillen part is covered by Theorem 3.1 and [FLY08, Theorem 5.4]. For $A(X / S)$, we compute

$$
\begin{aligned}
\log A(X / S)= & \frac{\chi\left(X_{\infty}\right)}{12} \log \left\|\eta_{X / S}\right\|_{L^{2}}^{2}+\frac{(-1)^{n+1}}{12}(f \circ b)_{*}\left(b^{*} \log \left(\left\|\eta_{X}\right\|^{2}\right) c_{n}(Q)\right) \\
& +\frac{(-1)^{n}}{12}(f \circ b)_{*}\left(b^{*} \log \left(\|d f\|^{2}\right) c_{n}(Q)\right) .
\end{aligned}
$$

The asymptotic of the first term is given by that of the $L^{2}$-metric, established in Proposition 2.3,

$$
-\log \left\|\eta_{X / S}\right\|_{L^{2}}^{2}=\alpha \log |s|^{2}-\beta\left(\left.\log |\log | s\right|^{2} \mid\right)+\text { continuous . }
$$

The second term and the third terms have asymptotics given by [Yos07, Lemma 4.4 and Corollary 4.6],

$$
\begin{aligned}
(f \circ b)_{*}\left(b^{*} \log \left(\left\|\eta_{X}\right\|^{2}\right) c_{n}(Q)\right) & =\left(\int_{b^{*} B} c_{n}(Q)\right) \log |s|^{2}+\text { continuous }, \\
(f \circ b)_{*}\left(b^{*} \log \left(\|d f\|^{2}\right) c_{n}(Q)\right) & =\left(\int_{E} c_{n}(Q)\right) \log |s|^{2}+\text { continuous } .
\end{aligned}
$$

For the first equality, we have used $\operatorname{div}\left(\eta_{X}\right)=B$, and for the second equality, we have used that the zero locus of $d f$ is exactly the singular locus $Z$ and $E=b^{-1}(Z)$. We obtain the final form by applying the formula $\int_{E} c_{n}(Q)=(-1)^{n}\left(\chi\left(X_{\infty}\right)-\chi\left(X_{0}\right)\right)$

The second part of the proposition is a consequence of the first. Indeed, by Theorem 3.1, it is enough to provide Mumford-good estimates on the continuous rests of the formulas above. But they are also as in Remark 3.2, by the same [Yos07, Lemma 4.4], and hence good in the sense of Mumford.

The third part is [FLY08, Theorem 4.9].

\subsection{Computation of $\alpha_{\mathrm{BCOV}}$}

The asymptotic formulas provided by [FLY08, Theorem 5.4] and Proposition 4.2 above are cumbersome, and the relation to topological invariants (for instance vanishing cycles) is not clear. We next show that several simplifications and cancellations occur in the expression defining $\alpha_{\mathrm{BCOV}}$. We rewrite it solely in terms of the characteristic classes $c_{n}(Q), c_{1}(Q) c_{n-1}(Q)$ and $c_{1}\left(b^{*} K_{X}\right) c_{n-1}(Q)$. We derive consequences for Kulikov-type families.

Recall that $b: X^{\prime} \rightarrow X$ denotes the Nash blowup of the morphism $f: X \rightarrow S$, with exceptional divisor $E$ and universal quotient bundle $Q$. We focus on the combination of characteristic 


\section{Metrics on Hodge BUndLes}

classes

$$
\omega:=\operatorname{Td}^{*}\left(\left.Q\right|_{E}\right) \sum_{p=0}^{n} \sum_{j=0}^{p} p(-1)^{j}\left(\operatorname{Td}^{*}\left(\left.\mathcal{O}(E)\right|_{E}\right)-\operatorname{ch}\left(\left.\mathcal{O}(E)\right|_{E}\right)^{p-j}\right) \operatorname{ch}\left(\left.b^{*} \Omega_{X}^{j}\right|_{E}\right) \cap\left[X^{\prime}\right] .
$$

To simplify the discussion, we remove the $\cap\left[X^{\prime}\right]$ from the notation. In the definition of $\alpha_{\mathrm{BCOV}}$, the class $\omega$ contributes through

$$
\int_{E} \frac{\omega}{c_{1}\left(\left.\mathcal{O}(E)\right|_{E}\right)}
$$

Because of the division by $c_{1}\left(\left.\mathcal{O}(E)\right|_{E}\right)$ and since $E$ is a divisor in $X^{\prime}$, we only seek a simple expression for the degree $n+1$ part of $\omega$. A priori, we know that this component has to be a multiple $c_{1}\left(\left.\mathcal{O}(E)\right|_{E}\right)$.

The starting point is to restrict the universal exact sequence

$$
0 \rightarrow L_{E} \rightarrow b^{*} \Omega_{X / S} \rightarrow Q \rightarrow 0
$$

to the exceptional divisor. Because $Q$ is locally free, the restriction of the sequence to $E$ remains exact. Moreover, we observe that $E$ lies above the singular locus $Z$ of the morphism $f: X \rightarrow S$, and hence $\left.b^{*} \Omega_{X / S}\right|_{E}=\left.b^{*} \Omega_{X}\right|_{E}$. Therefore, we obtain an exact sequence

$$
\left.\left.0 \rightarrow L_{E} \rightarrow b^{*} \Omega_{X}\right|_{E} \rightarrow Q\right|_{E} \rightarrow 0 .
$$

We also recall from Lemma 3.5 that $L_{E}$ is a line bundle on $E$ and that as bivariant classes with values in $A_{*}(E)$, the relation $c_{1}\left(L_{E}\right)=c_{1}\left(\mathcal{O}(E)_{\mid E}\right)$ holds. Taking exterior powers in (4.1) and replacing $c_{1}\left(L_{E}\right)$ by $c_{1}\left(\mathcal{O}(E)_{\mid E}\right)$, we find

$$
\operatorname{ch}\left(\left.b^{*} \Omega_{X}^{j}\right|_{E}\right)=\operatorname{ch}\left(\left.\Lambda^{j} Q\right|_{E}\right)+\operatorname{ch}\left(\left.\Lambda^{j-1} Q\right|_{E}\right) \operatorname{ch}\left(\left.\mathcal{O}(E)\right|_{E}\right),
$$

with the convention $\Lambda^{j-1} Q=0$ for $j=0$. From now on, to lighten the notation, we also remove the restriction to $E$ from the notation, by saying instead that a given relation holds on $E$. Therefore, on $E$, we can write $\omega=\vartheta+\vartheta^{\prime}$, where

$$
\vartheta=\left(\operatorname{Td}^{*}(Q)\right)\left(\operatorname{Td}^{*} \mathcal{O}(E)\right) \sum_{0 \leqslant j \leqslant p \leqslant n} p(-1)^{j}\left(\operatorname{ch}\left(\Lambda^{j} Q\right)+\operatorname{ch}\left(\Lambda^{j-1} Q\right) \operatorname{ch}(\mathcal{O}(E))\right)
$$

and the class $\vartheta^{\prime}$ is defined to be the rest. Actually, after a simple telescopic sum, $\vartheta^{\prime}$ simplifies to

$$
\vartheta^{\prime}=-\operatorname{Td}^{*}(Q) \sum_{p=0}^{n} p(-1)^{p} \operatorname{ch}\left(\Lambda^{p} Q\right)
$$

We now work on the class $\omega$.

LEMma 4.3. The class $\vartheta$ is the sum of three contributions

$$
\begin{aligned}
& \vartheta_{1}=-\frac{n(n+1)}{2}(-1)^{n} c_{n}(Q) c_{1}(\mathcal{O}(E))+\text { hct }, \\
& \vartheta_{2}=-\operatorname{Td}^{*}(Q) \operatorname{Td}^{*}(\mathcal{O}(E)) \sum_{j=0}^{n}(-1)^{j+1} \frac{j(j+1)}{2} \operatorname{ch}\left(\Lambda^{j} Q\right) \operatorname{ch}(\mathcal{O}(E)), \\
& \vartheta_{3}=-\operatorname{Td}^{*}(Q) \operatorname{Td}^{*}(\mathcal{O}(E)) \sum_{j=0}^{n}(-1)^{j} \frac{j(j-1)}{2} \operatorname{ch}\left(\Lambda^{j} Q\right),
\end{aligned}
$$

where hct is a shortcut for "higher-codimension terms." 


\section{Eriksson, G. Freixas i Montplet and C. Mourougane}

Proof. The proof is elementary and relies on the property [Ful98, Example 3.2.5]

$$
\operatorname{Td}^{*}(Q) \sum_{p=0}^{n}(-1)^{p} \operatorname{ch}\left(\Lambda^{p} Q\right)=(-1)^{n} c_{n}(Q)
$$

and the power-series expansions of $\operatorname{Td}^{*}(\mathcal{O}(E))$ and $\operatorname{ch}(\mathcal{O}(E))$ in $c_{1}(\mathcal{O}(E))$.

The relation (4.2) and the expressions for the classes $\vartheta$ and $\vartheta^{\prime}$ motivate the following definition. Definition 4.4. For a vector bundle $F$ of rank $r$, we define

$$
\begin{aligned}
P(F) & =\mathrm{Td}^{*}(F) \sum_{p=0}^{r}(-1)^{p} \operatorname{ch}\left(\Lambda^{p} F\right) \quad\left(=(-1)^{r} c_{r}(F)\right), \\
P^{\prime}(F) & =\mathrm{Td}^{*}(F) \sum_{p=0}^{r}(-1)^{p} p \operatorname{ch}\left(\Lambda^{p} F\right), \\
P^{\prime \prime}(F) & =\operatorname{Td}^{*}(F) \sum_{p=0}^{r}(-1)^{p} \frac{p(p-1)}{2} \operatorname{ch}\left(\Lambda^{p} F\right) .
\end{aligned}
$$

As the notation suggests, the classes $P^{\prime}(F)$ and $P^{\prime \prime}(F)$ are to be seen as the first and second derivatives of $P(F)$. More precisely, the following holds.

Lemma 4.5. The classes $P, P^{\prime}$ and $P^{\prime \prime}$ satisfy

$$
\begin{aligned}
P(F \oplus G) & =P(F) P(G), \\
P^{\prime}(F \oplus G) & =P^{\prime}(F) P(G)+P(F) P^{\prime}(G), \\
P^{\prime \prime}(F \oplus G) & =P^{\prime \prime}(F) P(G)+P^{\prime}(F) P^{\prime}(G)+P(F) P^{\prime \prime}(G) .
\end{aligned}
$$

In particular, given line bundles $L_{1}, \ldots, L_{r}$, we have

$$
\begin{aligned}
P^{\prime}\left(L_{1} \oplus \cdots \oplus L_{r}\right) & =\sum_{i=1}^{r} P\left(L_{1}\right) \cdots P^{\prime}\left(L_{i}\right) \cdots P\left(L_{r}\right), \\
P^{\prime \prime}\left(L_{1} \oplus \cdots \oplus L_{r}\right) & =\sum_{1 \leqslant i<j \leqslant r} P\left(L_{1}\right) \cdots P^{\prime}\left(L_{i}\right) \cdots P^{\prime}\left(L_{j}\right) \cdots P\left(L_{r}\right) .
\end{aligned}
$$

Proof. The first part is an easy computation using the multiplicativity of $\mathrm{Td}^{*}$ with respect to direct sums of vector bundles and the multiplicativity of ch with respect to tensor products of vector bundles. The conclusion for direct sums of line bundles requires the observation $P^{\prime \prime}(L)=0$ for a line bundle $L$.

In terms of $P^{\prime}$ and $P^{\prime \prime}$, the classes $\vartheta^{\prime}$ and $\vartheta_{3}$ are

$$
\vartheta^{\prime}=-P^{\prime}(Q), \quad \vartheta_{3}=-\operatorname{Td}^{*}(\mathcal{O}(E)) P^{\prime \prime}(Q) .
$$

For $\vartheta_{2}$, an easy computation gives the string of equalities,

$$
\begin{aligned}
\vartheta_{2} & =\left(P^{\prime}(Q)+P^{\prime \prime}(Q)\right) \operatorname{ch}(\mathcal{O}(E)) \operatorname{Td}^{*}(\mathcal{O}(E)) \\
& =\left(P^{\prime}(Q)+P^{\prime \prime}(Q)\right) c_{1}(\mathcal{O}(E))+P^{\prime}(Q) \operatorname{Td}^{*}(\mathcal{O}(E))-\vartheta_{3}+\text { het } \\
& =P^{\prime}(Q)\left(\frac{1}{2} c_{1}(\mathcal{O}(E))+\frac{1}{12} c_{1}(\mathcal{O}(E))^{2}\right)+P^{\prime \prime}(Q) c_{1}(\mathcal{O}(E))-\vartheta_{3}-\vartheta^{\prime}+\text { hct } .
\end{aligned}
$$

To conclude, we thus have to extract the codimension $n-1$ and $n$ parts of the class $P^{\prime}(Q)$ and the codimension $n$ class of $P^{\prime \prime}(Q)$. 


\section{Metrics on Hodge Bundles}

Lemma 4.6. (i) For the first derivative class, we have

$$
P^{\prime}(Q)=(-1)^{n} c_{n-1}(Q)+(-1)^{n} \frac{n}{2} c_{n}(Q)+\text { het },
$$

(ii) For the second derivative class, we have

$$
P^{\prime \prime}(Q)^{(n)}=(-1)^{n} \frac{n(3 n-5)}{24} c_{n}(Q)+(-1)^{n} \frac{1}{12} c_{1}(Q) c_{n-1}(Q) .
$$

Proof. By Lemma 4.5 and the splitting principle, we can suppose that $Q$ splits into a direct sum of line bundles $L_{1}, \ldots, L_{n}$.

For the first item, we use the formula for $P^{\prime}\left(L_{1} \oplus \cdots \oplus L_{n}\right)$ in Lemma 4.5. For this, we recall $P\left(L_{i}\right)=-c_{1}\left(L_{i}\right)$ from (4.2) and observe that

$$
P^{\prime}\left(L_{i}\right)=P\left(L_{i}\right)-\mathrm{Td}^{*}\left(L_{i}\right)=-1-\frac{1}{2} c_{1}\left(L_{i}\right)-\frac{1}{12} c_{1}\left(L_{i}\right)^{2}+\text { het } .
$$

After an elementary computation, one concludes by taking into account

$$
c_{n}(Q)=c_{1}\left(L_{1}\right) \cdots c_{1}\left(L_{n}\right), \quad c_{n-1}(Q)=\sum_{i=1}^{n} c_{1}\left(L_{1}\right) \cdots \widehat{c_{1}\left(L_{i}\right)} \cdots c_{1}\left(L_{n}\right) .
$$

For the second item, we proceed similarly. We first compute

$$
P^{\prime}\left(L_{i}\right) P^{\prime}\left(L_{j}\right)=\frac{1}{4} c_{1}\left(L_{i}\right) c_{1}\left(L_{j}\right)+\frac{1}{12} c_{1}\left(L_{i}\right)^{2}+\frac{1}{12} c_{1}\left(L_{j}\right)^{2}+\text { het . }
$$

Hence, we obtain

$$
\begin{aligned}
P^{\prime \prime}(Q)^{(n)}= & (-1)^{n} \frac{n(n-1)}{8} c_{n}(Q) \\
& +(-1)^{n} \frac{1}{12} \sum_{i<j} c_{1}\left(L_{1}\right) \cdots c_{1}\left(L_{i}\right)^{2} \cdots \widehat{c_{1}\left(L_{j}\right)} \cdots c_{n}\left(L_{n}\right) \\
& +(-1)^{n} \frac{1}{12} \sum_{i<j} c_{1}\left(L_{1}\right) \cdots \widehat{c_{1}\left(L_{i}\right)} \cdots c_{1}\left(L_{j}\right)^{2} \cdots c_{1}\left(L_{n}\right) .
\end{aligned}
$$

But we observe

$$
\begin{aligned}
\sum_{i<j} & c_{1}\left(L_{1}\right) \cdots c_{1}\left(L_{i}\right)^{2} \cdots \widehat{c_{1}\left(L_{j}\right)} \cdots c_{n}\left(L_{n}\right)+\widehat{\sum_{i<j}} c_{1}\left(L_{1}\right) \cdots \widehat{c_{1}\left(L_{i}\right)} \cdots c_{1}\left(L_{j}\right)^{2} \cdots c_{1}\left(L_{n}\right) \\
& =\left(c_{1}\left(L_{1}\right)+\cdots+c_{1}\left(L_{n}\right)\right) \sum_{i=1}^{n} c_{1}\left(L_{1}\right) \cdots \widehat{c_{1}\left(L_{i}\right)} \cdots c_{1}\left(L_{n}\right)-n c_{1}\left(L_{1}\right) \cdots c_{1}\left(L_{n}\right) \\
& =c_{1}(Q) c_{n-1}(Q)-n c_{n}(Q) .
\end{aligned}
$$

All in all, we conclude

$$
P^{\prime \prime}(Q)^{(n)}=(-1)^{n} \frac{n(3 n-5)}{24} c_{n}(Q)+(-1)^{n} \frac{1}{12} c_{1}(Q) c_{n-1}(Q) .
$$

Proposition 4.7. The class $\omega$ satisfies

$$
\int_{E} \frac{\omega}{c_{1}\left(\left.\mathcal{O}(E)\right|_{E}\right)}=(-1)^{n+1} \frac{9 n^{2}+11 n}{24} \int_{E} c_{n}(Q)+\frac{(-1)^{n}}{12} \int_{E} b^{*} c_{1}\left(K_{X}\right) c_{n-1}(Q) .
$$

Proof. We collect the identities in Lemma 4.3, the expression (4.3) for $\vartheta_{2}$ and the values provided by (4.1). We then observe that $c_{1}\left(\left.\mathcal{O}(E)\right|_{E}\right)+c_{1}\left(\left.Q\right|_{E}\right)=c_{1}\left(\left.b^{*} K_{X}\right|_{E}\right)$, as follows from (4.1) and $c_{1}\left(L_{E}\right)=c_{1}\left(\left.\mathcal{O}(E)\right|_{E}\right)$. This concludes the proof. 


\section{Eriksson, G. Freixas i Montplet and C. Mourougane}

Corollary 4.8. Suppose that $K_{X}$ is trivial on the singular locus $Z$. Then

$$
\int_{E} \frac{\omega}{c_{1}\left(\left.\mathcal{O}(E)\right|_{E}\right)}=-\frac{9 n^{2}+11 n}{24}\left(\chi\left(X_{\infty}\right)-\chi\left(X_{0}\right)\right) .
$$

In particular, if $f$ has isolated singularities, then

$$
\int_{E} \frac{\omega}{c_{1}\left(\left.\mathcal{O}(E)\right|_{E}\right)}=(-1)^{n+1} \frac{9 n^{2}+11 n}{24} \sum_{x \in X_{0}} \mu_{X_{0}, x} .
$$

Proof. By applying the projection formula, one infers

$$
\int_{E} b^{*} c_{1}\left(K_{X}\right) c_{n-1}(Q)=\int_{Z} c_{1}\left(K_{X}\right) b_{*} c_{n-1}(Q)
$$

By assumption, $K_{X}$ is trivial on $Z$, and hence this intersection number vanishes. We then apply the formula

$$
(-1)^{n} \int_{E} c_{n}(Q)=\chi\left(X_{\infty}\right)-\chi\left(X_{0}\right)
$$

In the case of isolated singularities, the difference of topological Euler characteristics is known to be the sum of the Milnor numbers (3.7) of the singularities. Precisely, we have

$$
\chi\left(X_{\infty}\right)-\chi\left(X_{0}\right)=(-1)^{n} \sum_{x \in X_{0}} \mu_{X_{0}, x} .
$$

This concludes the proof.

Corollary 4.9. The coefficient $\alpha_{\mathrm{BCOV}}$ is given by

$$
\alpha_{\mathrm{BCOV}}=-\frac{9 n^{2}+11 n+2}{24}\left(\chi\left(X_{\infty}\right)-\chi\left(X_{0}\right)\right)+\frac{\alpha}{12} \chi\left(X_{\infty}\right)+\frac{(-1)^{n}}{12} \int_{B} c_{n}\left(\Omega_{X / S}\right) .
$$

Proof. Notice that

$$
b^{*} c_{1}\left(K_{X}\right) c_{n-1}(Q) \cap[E]=c_{n-1}(Q) \cap c_{1}\left(b^{*} B\right) \cap[E]=c_{n-1}(Q) \cap c_{1}(E) \cap\left[b^{*} B\right]
$$

in the Chow group of the special fiber of $X^{\prime} \rightarrow S$. This is a consequence of the commutativity of intersection classes of Cartier divisors [Ful98, Section 2.4] and the definition of $c_{1}$ of a line bundle. Moreover, from Lemma 3.5(a), we have $c_{1}\left(L_{E}\right)=c_{1}\left(\mathcal{O}(E)_{\mid E}\right)$. Applying Chern classes on the tautological exact sequence on the Nash blowup, we deduce from the Whitney formula

$$
c_{n}\left(b^{*} \Omega_{X / S}\right) \cap\left[b^{*} B\right]=c_{n}(Q) \cap\left[b^{*} B\right]+c_{n-1}(Q) c_{1}(E) \cap\left[b^{*} B\right] .
$$

Observe that $c_{n}\left(b^{*} \Omega_{X / S}\right)=b^{*} c_{n}\left(\Omega_{X / S}\right)$ because $\Omega_{X / S}$ admits a two-term locally free resolution and $b$ is birational. Applying the projection formula, we finally find

$$
\int_{E} b^{*} c_{1}\left(K_{X}\right) c_{n-1}(Q)=\int_{B} c_{n}\left(\Omega_{X / S}\right)-\int_{b^{*} B} c_{n}(Q) .
$$

We finish the proof by plugging this relation into Proposition 4.7 and by the very definition of $\alpha_{\mathrm{BCOV}}$.

To sum up, we conclude by restating Proposition 4.2(a) for Kulikov families.

Theorem 4.10. Let $f: X \rightarrow S$ be a generically smooth family of Calabi-Yau varieties of dimension $n$, with a unique singular fiber of equation $s=0$. Assume that $X$ is a Kulikov family, in other words, that $B=\emptyset$ (for example, if $K_{X}$ is trivial). Choose a local holomorphic frame $\widetilde{\sigma}$ for the Kähler extension $\lambda_{\mathrm{BCOV}}\left(\widetilde{\Omega_{X / S}^{\bullet}}\right)$. 


\section{Metrics on Hodge Bundles}

Then the asymptotic of the BCOV norm of $\widetilde{\sigma}$ is

$$
\begin{aligned}
-\log \|\widetilde{\sigma}\|_{\mathrm{BCOV}}^{2}= & \alpha_{\mathrm{BCOV}} \log |s|^{2}-\left.\frac{\chi\left(X_{\infty}\right)}{12} \beta \log |\log | s\right|^{2} \mid+ \text { continuous } \\
= & {\left[-\frac{9 n^{2}+11 n+2}{24}\left(\chi\left(X_{\infty}\right)-\chi\left(X_{0}\right)\right)+\frac{\alpha}{12} \chi\left(X_{\infty}\right)\right] \log |s|^{2} } \\
& -\left.\frac{\chi\left(X_{\infty}\right)}{12} \beta \log |\log | s\right|^{2} \mid+ \text { continuous }
\end{aligned}
$$

where $\alpha$ and $\beta$ are as in Proposition 2.3.

Corollary 4.11. If $n \geqslant 2$ and $f: X \rightarrow S$ has only isolated ordinary quadratic singularities, then

$$
-\log \|\widetilde{\sigma}\|_{\mathrm{BCOV}}^{2}=(-1)^{n+1} \frac{9 n^{2}+11 n+2}{24} \# \operatorname{sing}\left(X_{0}\right) \log |s|^{2}+\text { continuous . }
$$

Proof. We observed in Section 2.1 that a Calabi-Yau degeneration with isolated singularities is automatically Kulikov. From Theorem 4.10 together with Corollary 2.9, Remark 2.10 and (4.4), we obtain that the dominant term is a weighted sum of Milnor numbers. For an isolated ordinary quadratic singularity, each such Milnor number is 1 .

\section{ACKNOWLEDGEMENTS}

The authors would like to thank Bo Berndtsson, Sébastien Boucksom, Lars Halvard Halle, Johannes Nicaise, Jan Stevens and Ken-Ichi Yoshikawa for interesting discussions and remarks. We thank the referee for his careful reading of our text. We also would like to extend our gratitude to the Hausdorff Institute for Mathematics in Bonn, where this project originated, for their support and hospitality, as well as the UMI of the CNRS in Montreal and the Max Planck Institute for Mathematics in Bonn.

\section{REFERENCES}

Abb00 A. Abbes, Cycles on arithmetic surfaces, Compos. Math. 122 (2000), no. 1, 23-111; https: //doi.org/10.1023/A:1001822419774.

Amb04 F. Ambro, Shokurov's boundary property, J. Differential Geom. 67 (2004), no. 2, 229-255; https://doi.org/10.4310/jdg/1102536201.

BB90 J.-M. Bismut and J.-B. Bost, Fibrés déterminants, métriques de Quillen et dégénérescence des courbes, Acta Math. 165 (1990), no. 1-2, 1-103; https://doi.org/10.1007/BF02391902.

BCOV94 M. Bershadsky, S. Cecotti, H. Ooguri and C. Vafa, Kodaira-Spencer theory of gravity and exact results for quantum string amplitudes, Comm. Math. Phys. 165 (1994), no. 2, 311-427; https://doi.org/10.1007/BF02099774.

Ber16 R. J. Berman, K-polystability of $\mathbb{Q}$-Fano varieties admitting Kähler-Einstein metrics, Invent. Math. 203 (2016), no. 3, 973-1025; https://doi.org/10.1007/s00222-015-0607-7.

BGS88a J.-M. Bismut, H. Gillet and C. Soulé, Analytic torsion and holomorphic determinant bundles. I. Bott-Chern forms and analytic torsion, Comm. Math. Phys. 115 (1988), no. 1, 49-78; https: //doi.org/10.1007/BF01238853.

BGS88b _ Analytic torsion and holomorphic determinant bundles. II. Direct images and BottChern forms, Comm. Math. Phys. 115 (1988), no. 1, 79-126; https://doi.org/10.1007/ BF01238854. 


\section{Eriksson, G. Freixas i Montplet and C. Mourougane}

BGS88c _ Analytic torsion and holomorphic determinant bundles. III. Quillen metrics on holomorphic determinants, Comm. Math. Phys. 115 (1988), no. 2, 301-351; https : //doi .org/10. $1007 / \mathrm{BF} 01466774$.

Bis97 J. M. Bismut, Quillen metrics and singular fibres in arbitrary relative dimension, J. Algebraic Geom. 6 (1997), no. 1, 19-149.

BJ17 S. Boucksom and M. Jonsson, Tropical and non-Archimedean limits of degenerating families of volume forms, J. Éc. Polytech. Math. 4 (2017), 87-139; https://doi.org/10.5802/jep. 39.

CK82 E. Cattani and A. Kaplan, Polarized mixed Hodge structures and the local monodromy of a variation of Hodge structure, Invent. Math. 67 (1982), no. 1, 101-115; https://doi.org/10. $1007 / \mathrm{BF} 01393374$.

Eri13 D. Eriksson, Degenerating Riemann surfaces and the Quillen metric, Int. Math. Res. Not. 2013 (2013), no. 2, 347-361; https://doi.org/10.1093/imrn/rnr234.

Eri16 _ Discriminants and Artin conductors, J. reine angew. Math. 712 (2016), 107-121; https://doi.org/10.1515/crelle-2014-0022.

FLY08 H. Fang, Z. Lu and K.-I. Yoshikawa, Analytic torsion for Calabi-Yau threefolds, J. Differential Geom. 80 (2008), no. 2, 175-259; https://doi.org/10.4310/jdg/1221066631.

FM00 O. Fujino and S. Mori, A canonical bundle formula, J. Differential Geom. 56 (2000), no. 1, 167-188; https://doi.org/10.4310/jdg/1090347529.

Ful98 W. Fulton, Intersection theory, second ed., Ergeb. Math. Grenzgeb. (3), vol. 2 (Springer-Verlag, Berlin, 1998); https://doi.org/10.1007/978-1-4612-1700-8.

GKZ08 I. M. Gelfand, M. M. Kapranov and A. V. Zelevinsky, Discriminants, resultants and multidimensional determinants, Modern Birkhäuser Classics (Birkhäuser Boston, Inc., Boston, MA, 2008); https://doi.org/10.1007/978-0-8176-4771-1.

HN12 L.H. Halle and J. Nicaise, Motivic zeta functions for degenerations of abelian varieties and Calabi-Yau varieties in Zeta Functions in Algebra and Geometry, Contemp. Math., vol. 566 (Amer. Math. Soc., Providence, RI, 2012), 233-259; https://doi.org/10.1090/conm/566/ 11223.

HN18_ Motivic zeta functions of degenerating Calabi-Yau varieties, Math. Ann. 370 (2018), no. 3, 1277-1320; https://doi.org/10.1007/s00208-017-1578-3.

Kaw82 Y. Kawamata, Kodaira dimension of algebraic fiber spaces over curves, Invent. Math. 66 (1982), no. 1, 57-71; https://doi.org/10.1007/BF01404756.

Kaw98 _ Subadjunction of log canonical divisors. II, Amer. J. Math. 120 (1998), no. 5, 893-899; https://doi.org/10.1353/ajm.1998.0038.

Kaw99___ Deformations of canonical singularities, J. Amer. Math. Soc. 12 (1999), no. 1, 85-92; https://doi.org/10.1090/S0894-0347-99-00285-4.

KM76 F.F. Knudsen and D. Mumford, The projectivity of the moduli space of stable curves. I. Preliminaries on "det" and "Div", Math. Scand. 39 (1976), no. 1, 19-55; https://doi.org/10. $7146 /$ math. scand.a-11642.

KN94 Y. Kawamata and Y. Namikawa, Logarithmic deformations of normal crossing varieties and smoothing of degenerate Calabi-Yau varieties, Invent. Math. 118 (1994), no. 3, 395-409; https: //doi.org/10.1007/BF01231538.

Kod64 K. Kodaira, On the structure of compact complex analytic surfaces. I, Amer. J. Math. 86 (1964), no. 4, 751-798; https://doi.org/10.2307/2373157.

Kol97 J. Kollár, Singularities of pairs, Algebraic Geometry - Santa Cruz 1995, Proc. Sympos. Pure Math., vol. 62 (Amer. Math. Soc., Providence, RI, 1997), 221-287.

Kol07_ Kodaira's canonical bundle formula and adjunction in Flips for 3-Folds and 4-Folds, Oxford Lecture Ser. Math. Appl., vol. 35 (Oxford Univ. Press, Oxford, 2007), 134-162; https: //doi.org/10.1093/acprof : oso/9780198570615.003.0008. 


\section{Metrics on Hodge Bundles}

KS04 K. Kato and T. Saito, On the conductor formula of Bloch, Publ. Math. Inst. Hautes Études Sci. (2004), no. 100, 5-151; https://doi.org/10.1007/s10240-004-0026-6.

Kul77 V.S. Kulikov, Degenerations of K3 surfaces and Enriques surfaces, Math. USSR Izv. 41 (1977), no. 5, 957-989; https://doi.org/10.1070/IM1977v011n05ABEH001753.

Lee10 N.-H. Lee, Calabi-Yau construction by smoothing normal crossing varieties, Internat. J. Math. 21 (2010), no. 6, 701-725; https://doi.org/10.1142/S0129167X10006173.

MT09 C. Mourougane and S. Takayama, Extension of twisted Hodge metrics for Kähler morphisms, J. Differential Geom. 83 (2009), no. 1, 131-161; https://doi.org/10.4310/jdg/1253804353.

Mum77 D. Mumford, Hirzebruch's proportionality theorem in the noncompact case, Invent. Math. 42 (1977), no. 1, 239-272; https://doi.org/10.1007/BF01389790.

Pie79 R. Piene, Ideals associated to a desingularization, Algebraic Geometry (Proc. Summer Meeting, Univ. Copenhagen, Copenhagen, 1978), Lecture Notes in Math., vol. 732 (Springer, Berlin, 1979), 503-517; https://doi.org/10.1007/BFb0066659.

RZ11 X. Rong and Y. Zhang, Continuity of extremal transitions and flops for Calabi-Yau manifolds (with Appendix B by M. Gross), J. Differential Geom. 89 (2011), no. 2, 233-269; https: //doi.org/10.4310/jdg/1324477411.

Sch73 W. Schmid, Variation of Hodge structure: the singularities of the period mapping, Invent. Math. 22 (1973), 211-319; https://doi.org/10.1007/BF01389674.

Ste77 J.H. M. Steenbrink, Mixed Hodge structure on the vanishing cohomology, Real and Complex Singularities (Proc. Ninth Nordic Summer School/NAVF Sympos. Math., Oslo, 1976) (Sijthoff and Noordhoff, Alphen aan den Rijn, 1977), 525-563.

Ste88 J. Stevens, On canonical singularities as total spaces of deformations, Abh. Math. Sem. Univ. Hamburg 58 (1988), 275-283; https://doi.org/10.1007/BF02941384.

Tei74 B. Teissier, Cycles évanescents, sections planes et conditions de Whitney, Astérisque 7-8 (1974), 285-362.

Tos15 V. Tosatti, Families of Calabi-Yau manifolds and canonical singularities, Int. Math. Res. Not. 2015 (2015), no. 20, 10586-10594; https://doi.org/10.1093/imrn/rnv001.

Wan97 C.-L. Wang, On the incompleteness of the Weil-Petersson metric along degenerations of CalabiYau manifolds, Math. Res. Lett. 4 (1997), no. 1, 157-171; https://doi.org/10.4310/MRL. 1997.v4.n1.a14.

Yos98 K.-I. Yoshikawa, Smoothing of isolated hypersurface singularities and Quillen metrics, Asian J. Math. 2 (1998), no. 2, 325-344; https://doi.org/10.4310/AJM.1998.v2.n2.a5.

Yos07 On the singularity of Quillen metrics, Math. Ann. 337 (2007), no. 1, 61-89; https: //doi.org/10.1007/s00208-006-0027-5.

Yos10_, Singularities and analytic torsion, 2010, arXiv:1007.2835.

Dennis Eriksson dener@chalmers.se

Department of Mathematics, Chalmers University of Technology and Gothenburg University, 41296 Göteborg, Sweden

Gerard Freixas i Montplet gerard.freixas@imj-prg.fr

C.N.R.S., Institut de Mathématiques de Jussieu, Paris Rive Gauche, 4, Place Jussieu 75005 Paris, France

Christophe Mourougane christophe.mourougane@univ-rennes1.fr

Institut de Recherche Mathématique de Rennes (IRMAR), Campus de Beaulieu, 35042 Rennes cedex, France 